\title{
THE TOROIDAL EMBEDDING ARISING FROM AN IRRATIONAL FAN
}

\author{
T. J. FORD
}

\begin{abstract}
A toroidal embedding is defined which does not assume the fan consists of rational cones. For a rational fan, the toroidal embedding is the usual toric variety. If the fan is not rational, the toroidal embedding is in general a quasicompact noetherian locally ringed space which is not a scheme. A divisor theory exists and a class group is defined. A second construction is also carried out which mimics the gluing construction of the usual toric variety, but which makes no reference to a lattice. The resulting scheme is separated but infinite dimensional. The Picard group is described in terms of the group of real valued locally linear support functions on the fan and the Brauer group is shown to be trivial. Many examples are given throughout the paper; in particular, it is shown that there is associated to a real hyperplane arrangement of full rank a toroidal embedding.
\end{abstract}

\section{INTRODUCTION}

Associated to the $\mathbb{Z}$-lattice $N$ is the algebraic torus $T_{N}$ whose coordinate ring is $k\left[x_{1}, x_{1}^{-1}, \ldots, x_{n}, x_{n}^{-1}\right]$, where $k$ is a field. Associated to a fan $\Delta$ on $N_{\mathbb{R}}$ consisting of finitely many strictly convex rational polyhedral cones is the toric variety $T_{N} \operatorname{emb}(\Delta)$. Our objective is to consider this construction when the fan $\Delta$ is not rational.

Throughout, $k$ is an algebraically closed field. As a standard reference we attempt to follow [13].

Let $\Delta$ be a fan on $N_{\mathbb{R}}$. That is, $\Delta$ is a finite set of strictly convex polyhedral cones satisfying: if $\tau \in \Delta$, then every face of $\tau$ is in $\Delta$ and if $\tau$ and $\sigma$ are in $\Delta$, then $\tau \cap \sigma$ is a common face of $\tau$ and $\sigma$. Define a topology on $\Delta$ under which the open sets are the subfans of $\Delta$. This defines a functor [5]

$$
\text { (finite fans on } \left.N_{\mathbb{R}}\right) \stackrel{\mathfrak{T}}{\rightarrow} \text { (finite top. spaces). }
$$

Let $\sigma$ be a strictly convex polyhedral cone in $N_{\mathbb{R}}$. Then $\sigma=\mathbb{R}_{\geq 0} \eta_{1}+\cdots+\mathbb{R}_{\geq 0} \eta_{r}$ for some minimal spanning set $\left\{\eta_{1}, \ldots, \eta_{r}\right\} \subseteq N_{\mathbb{R}}$. We say $\sigma$ is rational if there is a spanning set for $\sigma$ in $N$. Set $M=\operatorname{Hom}_{\mathbb{Z}}(N, \mathbb{Z})$. Let $\mathcal{S}_{\sigma}$ denote the semigroup $\{m \in M \mid\langle m, x\rangle \geq 0$ for all $x \in \sigma\}$. Our basic objects of study are the commutative semigroup rings $k\left[\mathcal{S}_{\sigma}\right]$. The ring $k\left[\mathcal{S}_{\sigma}\right]$ is noetherian if and only if $\sigma$ is rational (Proposition 2.1).

In Section 2 we show how to construct a ringed space $T_{N} \operatorname{emb}(\Delta)$ depending only on $k, \Delta$ and $N$ with the following properties. As a topological space, the point set $T_{N} \operatorname{emb}(\Delta)$ consists of the local rings at the prime ideals of the various $k\left[\mathcal{S}_{\sigma}\right]$ as $\sigma$ runs through the cones in $\Delta$ together with the Zariski topology.

Date: February 23, 2007.

1991 Mathematics Subject Classification. Primary 14M25; Secondary 20M25, 05B35.

Key words and phrases. Toroidal embedding, toric variety, hyperplane arrangement, Picard group, Brauer group. 
The underlying topological space of $T_{N} \operatorname{emb}(\Delta)$ is noetherian and quasi-compact. There is a natural embedding of $T_{N}$ as an open subset of $T_{N} \mathrm{emb}(\Delta)$. The group law on the $k$-rational points of $T_{N}$ extends to an action on the $k$-rational points of $T_{N} \operatorname{emb}(\Delta)$. The orbits correspond to the cones $\sigma$ in $\Delta$. Let $X=T_{N} \operatorname{emb}(\Delta)$ and $\tilde{X}$ the orbit space with the topology inherited from $X$. Then $\tilde{X}$ is homeomorphic to $\mathfrak{T}(\Delta)$. If $\Delta$ consists of rational cones, then $T_{N} \operatorname{emb}(\Delta)$ is the usual toric variety. If $\Delta$ is irrational, then $T_{N} \operatorname{emb}(\Delta)$ is a locally ringed space but in general does not have a covering by open affine subsets hence is not a scheme. The assignment $\Delta \mapsto T_{N} \operatorname{emb}(\Delta)$ is functorial. As an application, in Section 2.4 we associate to any hyperplane arrangement $\mathcal{A}$ of full rank in $\mathbb{R}^{l}$, a toroidal embedding $T_{N} \operatorname{emb}(\mathcal{A})$ which is $T_{N} \operatorname{emb}(\Phi(\mathcal{A}))$ for a lattice $N$ and fan $\Phi(\mathcal{A})$. In Section 3 we consider the theory of divisors on $T_{N} \operatorname{emb}(\Delta)$ and define a group of divisor classes which generalizes the class group of Weil divisors for toric varieties.

The extension of groups $M \hookrightarrow M_{\mathbb{R}}$ induces an extension of group algebras $k[M] \hookrightarrow k\left[M_{\mathbb{R}}\right]$ which induces a morphism of group schemes Spec $k\left[M_{\mathbb{R}}\right] \rightarrow T_{N}$. In Section 4 we define a scheme $X(\Delta)$ which has a natural action by the group scheme Spec $k\left[M_{\mathbb{R}}\right]$ and show that there exists a morphism of ringed spaces $X(\Delta) \rightarrow$ $T_{N} \operatorname{emb}(\Delta)$ which is equivariant with respect to the group actions. The Picard group of $X(\Delta)$ is described as a quotient of the group of real-valued $\Delta$-linear support functions on $|\Delta|$. We conjecture that this Picard group is trivial for a general complete fan on $\mathbb{R}^{3}$ such that every three-dimensional cone in $\Delta$ is non-simplicial. We show that every Azumaya algebra on $X(\Delta)$ is split, hence the Brauer group is trivial.

\section{IRRATIONAL FANS}

We first consider the case where the fan $\Delta=\Delta(\sigma)$ consists of one cone $\sigma$ together with all of its faces. Some properties of the semigroup ring associated to $\sigma$ are proved. In particular we consider the valuation of rank one that is defined by a cone of dimension one. The toroidal embedding is defined and we include several examples.

2.1. Irrational cones. In this section $N$ is a $\mathbb{Z}$-lattice of rank $n$ and $\Delta$ is a fan on $N_{\mathbb{R}}$. Suppose $\Delta$ is irrational. Then there will be some cone $\sigma \in \Delta$ which is strictly convex polyhedral but not rational. The usual construction of an affine toric variety for $\sigma$ fails in this case because the resulting ring is not noetherian. Let us outline the construction to see what happens. Suppose $\sigma$ is a strictly convex polyhedral cone in $N_{\mathbb{R}}$. Set $M=\operatorname{Hom}_{\mathbb{Z}}(N, \mathbb{Z})$. Form the dual cone $\sigma=\left\{y \in M_{\mathbb{R}} \mid\langle y, x\rangle \geq\right.$ 0 for all $x \in \sigma\}$. Set $\mathcal{S}_{\sigma}=M \cap \tilde{\sigma}$. Then $\mathcal{S}_{\sigma}$ is a submonoid of $M$. Form the commutative semigroup ring $k\left[\mathcal{S}_{\sigma}\right]$.

Proposition 2.1. If $\sigma$ is a strictly convex polyhedral cone in $N_{\mathbb{R}}$, then the following are equivalent.

(1) $\sigma$ is rational.

(2) The monoid $\mathcal{S}_{\sigma}$ is finitely generated.

(3) The ring $k\left[\mathcal{S}_{\sigma}\right]$ is a noetherian integrally closed domain.

(4) The ring $k\left[\mathcal{S}_{\sigma}\right]$ is a Krull domain.

Proof. (1) implies (2): If $\sigma$ is rational, then this is Gordan's lemma [13, p. 3].

(2) implies (1): If $\mathcal{S}_{\sigma}$ is finitely generated, then let $\left\{m_{1}, \ldots, m_{n}\right\}$ be a generating set. Let $\tau=\sum \mathbb{R}_{\geq 0} m_{i}$. Then $\tau$ is a convex cone contained in $\sigma$ and contains all of 
the lattice points of $\check{\sigma}$. We show that the two cones are equal, hence $\sigma$ is rational. Now $\check{\sigma}$ and $\tau$ are both closed in $M_{\mathbb{R}}$. Suppose $\check{\sigma} \neq \tau$. Then there is a nonempty open $U \subseteq \check{\sigma}-\tau$. But $U$ contains a rational point of $M_{\mathbb{R}}$ and since $\sigma-\tau$ is a cone, there is a lattice point in $\sigma-\tau$ which is a contradiction.

(2) implies (3) and (3) implies (4) are both routine.

(4) implies (2): Follows from [7, Theorem 15.6 and Theorem 15.11].

Let $\sigma$ be a strictly convex polyhedral cone in $N_{\mathbb{R}}$. We see in Section 2.3 below that the ring $k\left[\mathcal{S}_{\sigma}\right]$ is an integrally closed integral domain. When $\sigma$ is irrational and has dimension two in $\mathbb{R}^{2}$, the next result shows that $k\left[\mathcal{S}_{\sigma}\right]$ is not a unique factorization domain.

Proposition 2.2. Let $\sigma$ be a two-dimensional strictly convex polyhedral cone in $N_{\mathbb{R}}=\mathbb{R}^{2}$. If $\sigma$ is irrational, then $k\left[\mathcal{S}_{\sigma}\right]$ is not a unique factorization domain.

Proof. Following [13, Prop. 1.21, p. 26], let $\Theta$ be the convex hull in $M_{\mathbb{R}}$ of $(\sigma \curvearrowleft M)-$ $\{0\}$. The lattice points in $\Theta$ lying on the compact edges of the boundary polygon $\partial \Theta$ of $\Theta$ form a minimal set of generators of the semigroup $\mathcal{S}_{\sigma}$. This set is infinite, by Proposition 2.1. If we pick any three consecutive generators $k_{0}, k_{1}, k_{2}$ of this semigroup, then there exists an integer $c \geq 2$ such that [13, p. 26] $k_{0}+k_{2}=c k_{1}$. Therefore, in $k\left[\mathcal{S}_{\sigma}\right]$ there is the relation $\chi^{k_{0}} \chi^{k_{2}}=\left(\chi^{k_{1}}\right)^{c}$. But $\chi^{k_{0}}, \chi^{k_{2}}$, and $\chi^{k_{1}}$ are irreducible elements of $k\left[\mathcal{S}_{\sigma}\right]$.

If $\sigma$ is a strictly convex polyhedral cone in $N_{\mathbb{R}}$, let $\operatorname{Supp}_{\mathbb{Q}}(\sigma)$ denote the smallest $\mathbb{R}$-subspace of $N_{\mathbb{R}}$ satisfying

(1) $\sigma \subseteq \operatorname{Supp}_{\mathbb{Q}}(\sigma)$, and

(2) $\operatorname{Supp}_{\mathbb{Q}}(\sigma)$ has a $\mathbb{Q}$-structure. That is, $\operatorname{Supp}_{\mathbb{Q}}(\sigma) \cap N_{\mathbb{Q}}$ contains an $\mathbb{R}$-basis for $\operatorname{Supp}_{\mathbb{Q}}(\sigma)$.

Lemma 2.3. If $\sigma$ is a strictly convex polyhedral cone in $N_{\mathbb{R}}$, then

$$
\operatorname{Supp}_{\mathbb{Q}}(\sigma)^{\perp} \cap M_{\mathbb{Q}}=\sigma^{\perp} \cap M_{\mathbb{Q}} .
$$

Proof. Since $\operatorname{Supp}_{\mathbb{Q}}(\sigma) \supseteq \sigma$, it follows that $\operatorname{Supp}_{\mathbb{Q}}(\sigma)^{\perp} \subseteq \sigma^{\perp}$ and $\operatorname{Supp}_{\mathbb{Q}}(\sigma)^{\perp} \cap M_{\mathbb{Q}} \subseteq$ $\sigma^{\perp} \cap M_{\mathbb{Q}}$. For the other containment, let $C$ denote $\sigma^{\perp} \cap M_{\mathbb{Q}}$. Then $C$ is a $\mathbb{Q}$ subspace of $M_{\mathbb{R}}$ and since $C \subseteq \sigma^{\perp}$, it follows that $C^{\perp} \supseteq\left(\sigma^{\perp}\right)^{\perp}$. Now $\sigma \subseteq C^{\perp}$ and $C^{\perp}$ is an $\mathbb{R}$-subspace of $N_{\mathbb{R}}$ with a $\mathbb{Q}$-structure. So $C^{\perp} \supseteq \operatorname{Supp}_{\mathbb{Q}}(\sigma)$. Since $C_{\mathbb{R}} \subseteq\left(C^{\perp}\right)^{\perp} \subseteq \operatorname{Supp}_{\mathbb{Q}}(\sigma)^{\perp}$, and $C_{\mathbb{R}} \cap M_{\mathbb{Q}}=\sigma^{\perp} \cap M_{\mathbb{Q}}$, it follows that $\sigma^{\perp} \cap M_{\mathbb{Q}} \subseteq$ $\operatorname{Supp}_{\mathbb{Q}}(\sigma)^{\perp} \cap M_{\mathbb{Q}}$.

Proposition 2.4. If $\sigma$ is a strictly convex polyhedral cone in $N_{\mathbb{Q}}$ with $\mathcal{S}_{\sigma}=\sigma \curvearrowleft M$, and $s=\operatorname{dim}_{\mathbb{R}} \operatorname{Supp}_{\mathbb{Q}}(\sigma)$, then

(1) the group of invertible elements of $\mathcal{S}_{\sigma}$ is a free $\mathbb{Z}$-module of rank $n-s$,

(2) the Picard group, Pic $k\left[\mathcal{S}_{\sigma}\right]$, is trivial, and

(3) the Brauer group, $\mathrm{B}\left(k\left[\mathcal{S}_{\sigma}\right]\right)$, is isomorphic to $\mathrm{B}\left(T^{n-s}\right) \cong(\mathbb{Q} / \mathbb{Z})^{\left(\frac{n-s}{2}\right)}$, the Brauer group of the $(n-s)$-dimensional torus Spec $k\left[\sigma^{\perp} \cap M\right]$.

Proof. The group of invertible elements of $\mathcal{S}_{\sigma}$ is equal to the set $\sigma^{\perp} \cap M$ and applying Lemma 2.3 gives $\operatorname{rank}_{\mathbb{Z}}\left(\sigma^{\perp} \cap M\right)=\operatorname{dim}_{\mathbb{Q}}\left(\sigma^{\perp} \cap M_{\mathbb{Q}}\right)=$ $\operatorname{dim}_{\mathbb{Q}}\left(\operatorname{Supp}_{\mathbb{Q}}(\sigma)^{\perp} \cap M_{\mathbb{Q}}\right)=\operatorname{dim}_{\mathbb{R}}\left(\operatorname{Supp}_{\mathbb{Q}}(\sigma)^{\perp}\right)=n-\operatorname{dim}_{\mathbb{R}}\left(\operatorname{Supp}_{\mathbb{Q}}(\sigma)\right)$.

If $\sigma$ is rational, then by [2, Corollary 10] $\operatorname{Pic} k\left[\mathcal{S}_{\sigma}\right] \cong \operatorname{Pic} T^{q}$ and $\mathrm{B}\left(k\left[\mathcal{S}_{\sigma}\right]\right) \cong$ $\mathrm{B}\left(T^{q}\right)$ where $T^{q}$ is the $q$-dimensional torus and $q$ is the codimension of $\sigma$ in $N_{\mathbb{R}}$. 
But $\operatorname{Pic} T^{q}=0$ and $\mathrm{B}\left(T^{q}\right)=(\mathbb{Q} / \mathbb{Z})^{\left(\begin{array}{l}q \\ 2\end{array}\right)}$. If $\sigma$ is irrational, then we can find a strictly descending sequence of rational cones $\left\{\sigma_{i}\right\}_{i \geq 1}$ in $N_{\mathbb{R}}$ such that $\sigma \subseteq \sigma_{i}$ for each $i$ and $\sigma=\bigcap_{i>1} \sigma_{i}$. Then we have an ascending chain of rings $k\left[\mathcal{S}_{\sigma_{1}}\right] \subseteq k\left[\mathcal{S}_{\sigma_{2}}\right] \subseteq \cdots \subseteq$ $k\left[\mathcal{S}_{\sigma}\right]$ such that $k\left[\mathcal{S}_{\sigma}\right]=\bigcup_{i \geq 1} k\left[\mathcal{S}_{\sigma_{i}}\right]$. By the proof for the rational case, Pic $k\left[\mathcal{S}_{\sigma_{i}}\right]=$ 0 for each $i$. Given an invertible module $L$ over $k\left[\mathcal{S}_{\sigma}\right]$, by the usual finiteness argument, there is an $i$ and invertible module $L_{i}$ over $k\left[\mathcal{S}_{\sigma_{i}}\right]$ such that $L=L_{i} \otimes$ $k\left[\mathcal{S}_{\sigma}\right]$. But $L_{i}$ is free, hence so is $L$. Likewise, given an Azumaya $k\left[\mathcal{S}_{\sigma}\right]$-algebra, there is an $i$ and an Azumaya $k\left[\mathcal{S}_{\sigma_{i}}\right]$-algebra $A_{i}$ such that $A=A_{i} \otimes_{k\left[\mathcal{S}_{\sigma_{i}}\right]} k\left[\mathcal{S}_{\sigma}\right]$. By the result for the rational case we can assume $A_{i}$ is an Azumaya $k\left[\sigma_{i}^{\perp} \cap M\right]$-algebra. But $\sigma_{i}^{\perp} \subseteq \sigma^{\perp}$, hence $A$ is an Azumaya $k\left[\sigma^{\perp} \cap M\right]$-algebra.

Example 2.5. Suppose $\sigma=\mathbb{R}_{\geq 0} \eta$ is a one-dimensional cone in $N_{\mathbb{R}}=\mathbb{R}^{3}$. If $\eta=$ $\left(\begin{array}{c}\sqrt{2} \\ 1 \\ 2\end{array}\right)$, then $\operatorname{Supp}_{\mathbb{Q}}(\sigma)$ is spanned by the set $\left\{\left(\begin{array}{l}1 \\ 0 \\ 0\end{array}\right),\left(\begin{array}{l}0 \\ 1 \\ 2\end{array}\right)\right\}$. So the group of invertible elements of $\mathcal{S}_{\sigma}$ has rank one and $\mathrm{B}\left(k\left[\mathcal{S}_{\sigma}\right]\right)$ is trivial.

Example 2.6. Suppose $\sigma=r_{0}+r_{1}$ is a two-dimensional cone in $N_{\mathbb{R}}=\mathbb{R}^{3}$ where $r_{i}=\mathbb{R}_{\geq 0} \eta_{i}$.

(1) If $\eta_{0}=\left(\begin{array}{l}1 \\ 0 \\ 0\end{array}\right)$ and $\eta_{1}=\left(\begin{array}{l}\sqrt{2} \\ \sqrt{3} \\ \sqrt{5}\end{array}\right)$, then $\operatorname{Supp}_{\mathbb{Q}}(\sigma)=N_{\mathbb{R}}$ and the group of invertible elements of $\mathcal{S}_{\sigma}$ has rank zero.

(2) If $\eta_{0}=\left(\begin{array}{l}1 \\ 0 \\ 0\end{array}\right)$ and $\eta_{1}=\left(\begin{array}{c}\sqrt{2} \\ a \\ b\end{array}\right)$, where $a, b$ are rational numbers, then $\operatorname{Supp}_{\mathbb{Q}}(\sigma)$ is spanned by the set $\left\{\left(\begin{array}{l}1 \\ 0 \\ 0\end{array}\right),\left(\begin{array}{l}0 \\ a \\ b\end{array}\right)\right\}$, and the group of invertible elements of $\mathcal{S}_{\sigma}$ has rank one.

(3) If $\eta_{0}=\left(\begin{array}{l}1 \\ 0 \\ 0\end{array}\right)$ and $\eta_{1}=\left(\begin{array}{c}a \\ \sqrt{2} \\ b\end{array}\right)$, where $a, b$ are rational numbers, then $\operatorname{Supp}_{\mathbb{Q}}(\sigma)$ is spanned by the set $\left\{\left(\begin{array}{l}1 \\ 0 \\ 0\end{array}\right),\left(\begin{array}{l}0 \\ 1 \\ 0\end{array}\right),\left(\begin{array}{l}0 \\ 0 \\ b\end{array}\right)\right\}$. If $b=0$, then we see that $\operatorname{dim}_{\mathbb{R}}\left(\operatorname{Supp}_{\mathbb{Q}}(\sigma)\right)=2$ and the group of invertible elements of $\mathcal{S}_{\sigma}$ has rank one. If $b \neq 0$, then we see that $\operatorname{dim}_{\mathbb{R}}\left(\operatorname{Supp}_{\mathbb{Q}}(\sigma)\right)=3$ and the group of invertible elements of $\mathcal{S}_{\sigma}$ has rank zero.

Remark 2.7. We list here some questions raised by the above discussion. Let $N=$ $\mathbb{Z}^{2}$, and $r_{1}, r_{2}, \sigma_{1}, \sigma_{2}$ be cones in $N_{\mathbb{R}}$ with $\operatorname{dim} \sigma_{i}=2$ and $\operatorname{dim} r_{i}=1$.

(a) Is $M \cap \sigma_{1} \cong M \cap \sigma_{2}$ as semigroups? If $\sigma_{1}$ or $\sigma_{2}$ is rational, this is false. How about the case where both are irrational?

(b) Is $M \cap r_{1} \cong M \cap r_{2}$ as semigroups? This is false if $r_{1}$ is rational and $r_{2}$ is irrational, but true if both are rational. How about if both are irrational? 
(c) Is there an automorphism $\theta$ of $M$ that induces an isomorphism in (b)? We give a counterexample where $r_{1}$ and $r_{2}$ are both irrational. Take

$$
r_{1}=\mathbb{R}_{\geq 0}\left(\begin{array}{c}
1 \\
\sqrt{2}
\end{array}\right) \text { and } r_{2}=\mathbb{R}_{\geq 0}\left(\begin{array}{c}
1 \\
\sqrt{3}
\end{array}\right) .
$$

Suppose such an automorphism $\theta$ exists. Then there is a positive real number $\lambda$ and integers $a, b, c$, and $d$ satisfying:

$$
\left(\begin{array}{ll}
a & b \\
c & d
\end{array}\right)\left(\begin{array}{c}
1 \\
\sqrt{2}
\end{array}\right)=\lambda\left(\begin{array}{c}
1 \\
\sqrt{3}
\end{array}\right) \text { and } a d-b c= \pm 1 .
$$

It is straightforward to eliminate $\lambda$ and see that there is no integer solution to (2.1).

2.2. The valuation given by an irrational cone of dimension 1. Let $\rho$ be a cone of dimension one in $N_{\mathbb{R}}$. Suppose $\rho=\mathbb{R}_{\geq 0} \eta$ for $\eta \in N_{\mathbb{R}}$. Set $\check{\rho}=\left\{y \in M_{\mathbb{R}} \mid\langle\eta, y\rangle \geq 0\right\}$ and $\mathcal{S}_{\rho}=\check{\rho} \cap M$. Upon restricting to lattice points, we can view $\eta$ as an element of $\operatorname{Hom}_{\mathbb{Z}}(M, \mathbb{R})$. In this case $\eta$ defines a valuation $v_{\rho}$ on $k(M)$. Up to equivalence, $v_{\rho}$ does not depend on the choice of $\eta$. The value group is $G_{\rho}=\{\langle\eta, m\rangle \mid m \in M\} \subseteq \mathbb{R}$ hence $v_{\rho}$ has rank one. Up to isomorphism, $G_{\rho}$ does not depend on the choice of $\eta$. The rational rank of $v_{\rho}$ is the rank of the free $\mathbb{Z}$-module $G_{\rho}$. The rational rank of $v_{\rho}$ is no greater than $n=\operatorname{rank}_{\mathbb{Z}}(N)$.

The next lemma adds another equivalent condition to the list of Proposition 2.1 in the case of cones of dimension one.

Lemma 2.8. The rational rank of $v_{\rho}$ is one if and only if $\rho$ is a rational cone.

Proof. Suppose $\eta$ is rational. Then we can assume $\eta$ is a lattice point and $G_{\rho} \cong \mathbb{Z}$. Suppose $G_{\rho}=\mathbb{Z} r$ for some $r \in \mathbb{R}_{>0}$. Then $\left\langle\eta, m_{0}\right\rangle=r$ for some $m_{0} \in M$. Replace $\eta$ with $\frac{1}{r} \eta$. Thus $G_{\rho}=\mathbb{Z}$ and $\left\langle\eta, m_{0}\right\rangle=1$. It follows that $\eta \in N$.

The valuation ring of $v_{\rho}$ is the local ring of $k\left[\mathcal{S}_{\rho}\right]$ at the maximal ideal generated by the monomials $\left\{\chi^{m} \mid m \in \mathcal{S}_{\rho}\right\}$. This ring is noetherian if and only if $\rho$ is rational [15, II, Theorem 16, p. 41]. Notice that $k\left[\mathcal{S}_{\rho}\right]$ is the subring of $k[M]$ consisting of elements $x$ with valuation $v_{\rho}(x) \geq 0$.

Example 2.9. Let $n=2, \eta=\left(\begin{array}{c}1 \\ \sqrt{2}\end{array}\right) \in N_{\mathbb{R}}$ and $\rho=\mathbb{R}_{\geq 0} \eta$. Then $G_{\rho}=\{a+\sqrt{2} b \mid a, b \in$ $\mathbb{Z}\}$ has rational rank two. The reader should compare [15, II, Example 1, p. 100].

2.3. The toroidal embedding defined by a fan on $N_{\mathbb{R}}$. We define $T_{N} \operatorname{emb}(\Delta)$ for $\Delta$ an irrational fan on $N_{\mathbb{R}}$. Given any $\sigma$ in $\Delta$, form the dual cone $\sigma=\left\{y \in M_{\mathbb{R}} \mid\langle x, y\rangle \geq\right.$ 0 for all $x \in \sigma\}$ in $M_{\mathbb{R}}$. Let $\mathcal{S}_{\sigma}=\sigma \curvearrowleft M$ be the monoid of lattice points in $\sigma$ and form the semigroup algebra $k\left[\mathcal{S}_{\sigma}\right]$. From the proof of [13, Prop. 1.1] it is possible to check that $\mathcal{S}_{\sigma}$ generates $M$ as a group and is integrally closed in $M$. Therefore $k\left[\mathcal{S}_{\sigma}\right]$ is a subring of $k[M]$ and they have the same quotient field, namely the field of rational functions on the torus $K=k(M)$. So $k\left[\mathcal{S}_{\sigma}\right]$ is an integrally closed integral domain (see [7, p. 152]). By [7, Theorem 21.4, p. 290] $\operatorname{dim} k\left[\mathcal{S}_{\sigma}\right]=\operatorname{dim} k[M]=\operatorname{rank}_{\mathbb{Z}} M$.

For the rest of the construction, we use the terminology of [15, Vol. II, chapter VI, section 17]. Let $L$ be the set of all quasi-local rings (noetherian or nonnoetherian) between $k$ and $K$. For $P$ in $L$, denote by $\mathfrak{m}(P)$ the unique maximal ideal of $P$. Define a topology on $L$ by the following rule. If $\mathfrak{d}$ is any ring between $k$ and $K$, denote by $L(\mathfrak{d})$ the subset of $L$ made up of all quasi-local rings $P$ containing $\mathfrak{d}$. Let $\mathfrak{d}$ range over all subrings of $K$ that are finitely generated over $k$, and 
take the family of corresponding sets $L(\mathfrak{d})$ as a basis for open sets in $L$. In this topology, the closure of the singleton set $\{P\} \subseteq L$ is the set of all quasi-local rings $P^{\prime}$ between $k$ and $P$. For any ring $\mathfrak{d}$ between $k$ and $K$, denote by $V(\mathfrak{d})$ the subset of $L$ consisting of all quasi-local rings $\mathfrak{d}_{P}$ where $P \in \operatorname{Spec} \mathfrak{d}-\{(0)\}$. By [15, Lemma 1 , p. 116] the mapping $f: V(\mathfrak{d}) \rightarrow \operatorname{Spec} \mathfrak{d}-\{(0)\}$ defined by $f(P)=\mathfrak{m}(P) \cap \mathfrak{d}$ is a homeomorphism. A subset $M$ of $L$ is said to be irredundant (resp. complete) if, for any valuation $v$ of $K / k$ (trivial or non-trivial), the valuation ring $R_{v}$ dominates at most one (resp. at least one) element of $M$. For noetherian integral schemes, the irredundant property translates into the valuative criterion for separatedness over $k$ (see [9, p. 97]). For any ring $\mathfrak{d}$ between $k$ and $K$, the subset $V(\mathfrak{d})$ is irredundant.

One thing that makes the above construction cumbersome is the absence of the field $K$ from the set $L$. From now on, we include $K$ as an element of $L$, hence there is now a generic point in $L$. In this case we can assume $K$ is also in $V(\mathfrak{d})$ and can identify $V(\mathfrak{d})$ with Spec $\mathfrak{d}$.

Definition 2.10. The toroidal embedding associated to the field $k$, the $\mathbb{Z}$-lattice $N$ and the fan $\Delta$ on $N_{\mathbb{R}}$ is the ringed space whose underlying topological space is

$$
T_{N} \operatorname{emb}(\Delta)=\bigcup_{\sigma \in \Delta} V\left(k\left[\mathfrak{S}_{\sigma}\right]\right) .
$$

The set $T_{N} \operatorname{emb}(\Delta)$ comes with a structure sheaf of rings $\mathcal{O}$, making it into a ringed space. Namely, to each open set $U$, assign

$$
\mathcal{O}(U)=\bigcap_{P \in U} P
$$

Since each point $x$ of $L$ is a quasi-local ring, we would like the local ring $\mathcal{O}_{x}$ to be simply $x$ itself. We see later that this is true (Lemma 2.20).

Note that $T_{N} \operatorname{emb}(\Delta)$ is not irredundant in general. For example, let $\sigma$ be an irrational two-dimensional cone in $N_{\mathbb{R}}$, where rank $N=2$. Assume both onedimensional faces of $\sigma$ are irrational. Let $\tau$ be a one-dimensional face of $\sigma$. Let $P_{\tau}$ denote the local ring at the maximal ideal of $k\left[\mathcal{S}_{\tau}\right]$ generated by all monomials. Likewise let $P_{\sigma}$ denote the local ring at the maximal ideal of $k\left[\mathfrak{S}_{\sigma}\right]$ generated by the monomials. Then $P_{\tau}$ dominates $P_{\sigma}$. From this example we see that $T_{N} \operatorname{emb}(\Delta(\sigma))$ cannot be a scheme in general. This is because $P_{\tau}$ is in every neighborhood of $P_{\sigma}$, hence $P_{\sigma}$ has no irredundant neighborhood. But $\operatorname{Spec} A$ is separated for any ring $A$. It follows that $T_{N} \mathrm{emb}(\Delta)$ is not a scheme in general.

Remark 2.11. If $\Delta$ is a complete fan on $N_{\mathbb{R}}$, is $T_{N} \operatorname{emb}(\Delta)$ a complete subset of $L$ ? In this case it is true that every rank one valuation ring $R_{v}$ of $K / k$ dominates at least one element of $T_{N} \mathrm{emb}(\Delta)$ (see [13, p. 17]). How about a general valuation?

Let $\sigma$ be a cone in $\Delta$. Let $I$ be the ideal in $k\left[\mathcal{S}_{\sigma}\right]$ generated by the set of monomials which are not invertible. That is, $I$ is generated by the set $\left\{\chi^{m} \mid m \in M\right.$ and $\left.\langle x, m\rangle\right\rangle$ 0 for some $x \in \sigma\}$. Define orb $\sigma$ to be the closed subset of Spec $k\left[\mathcal{S}_{\sigma}\right]$ consisting of those prime ideals that contain $I$. The semigroup $\mathcal{S}_{\sigma}$ decomposes into the subgroup $M \cap \sigma^{\perp}$ and the ideal $I_{1}=\{m \in M \mid\langle x, m\rangle>0$ for some $x \in \sigma\}$. The group $M \cap \sigma^{\perp}$ is finitely generated and torsion free since it is a subgroup of $M$. The ideal $I$ is generated by $\chi^{I_{1}}$. If $M \cap \sigma^{\perp}$ has rank $t$, then $k\left[\mathcal{S}_{\sigma}\right] / I$ is isomorphic to $k\left[x_{1}, x_{1}^{-1}, \ldots, x_{t}, x_{t}^{-1}\right]$. So orb $\sigma$ is an algebraic torus of dimension $t$, where $t$ is possibly equal to zero. Let $P_{\sigma}$ denote the quasi-local ring of $k\left[\mathcal{S}_{\sigma}\right]$ at the prime ideal $I$. Then $P_{\sigma}$ is the generic stalk of orb $\sigma$. 
Lemma 2.12. Let $\sigma$ be a maximal cone in $\Delta$. The closure of $P_{\sigma}$ in $T_{N} \operatorname{emb}(\Delta)$ is $\operatorname{orb} \sigma$, hence orb $\sigma$ is closed. Also orb $\sigma$ is disjoint from $V\left(k\left[\mathcal{S}_{\tau}\right]\right)$ for every $\tau$ different from $\sigma$. If $\sigma_{1}, \ldots, \sigma_{a}$ are all of the maximal cones of $\Delta$, then $\operatorname{orb} \sigma_{1}, \ldots, \operatorname{orb} \sigma_{a}$ are pairwise disjoint closed subsets of $T_{N} \operatorname{emb}(\Delta)$.

Proof. We prove the first statement and leave the rest to the reader. First note that the closure of $P_{\sigma}$ in $L$ consists of all quasi-local rings between $k$ and $P_{\sigma}$. If $\tau$ is any cone in $\Delta$ different from $\sigma$, then since $\sigma$ is maximal, $\sigma$ is not a face of $\tau$. Therefore there exists a lattice point $m$ in the relative interior of $\tau$ such that $m$ is not in $\tilde{\sigma}$. Let $y=\chi^{m}$ and consider $L(k[y])$. Since $y \notin P_{\sigma}, P_{\sigma} \notin L(k[y])$. Since $k[y] \subseteq k\left[\mathcal{S}_{\tau}\right]$, $P_{\sigma}$ is not a localization of $k\left[\mathcal{S}_{\tau}\right]$. So the closure of $P_{\sigma}$ is the subset of $V\left(k\left[\mathcal{S}_{\sigma}\right]\right)$ corresponding to those primes that contain $I$, which is $\operatorname{orb} \sigma$.

Proposition 2.13. If the fan $\Delta$ consists of cones of dimension less than or equal to one, then $T_{N} \operatorname{emb}(\Delta)$ is a scheme.

Proof. If $\Delta(1)$ is empty, then $T_{N} \operatorname{emb}(\Delta)=T_{N}$. Otherwise there is an open affine covering $\left\{V\left(k\left[\mathcal{S}_{\sigma}\right]\right) \mid \sigma \in \Delta(1)\right\}$.

Since the subset Spec $k\left[\mathcal{S}_{\sigma}\right]=V\left(k\left[\mathcal{S}_{\sigma}\right]\right) \subseteq T_{N} \operatorname{emb}(\Delta)$ is not open in general, we want to attach some extra points to $V\left(k\left[\mathcal{S}_{\sigma}\right]\right)$. We attach any point $P \in T_{N} \operatorname{emb}(\Delta)$ that dominates some $P^{\prime} \in V\left(k\left[\mathcal{S}_{\sigma}\right]\right)$. Set

$$
U_{\sigma}=\bigcup_{\tau \leq \sigma} \operatorname{orb} \tau
$$

Notice that $U_{\sigma}=T_{N} \operatorname{emb}(\Delta(\sigma))$.

Lemma 2.14. For any $\sigma \in \Delta$, the subset $U_{\sigma} \subseteq T_{N} \operatorname{emb}(\Delta)$ is open. Therefore $\left\{U_{\sigma} \mid \sigma \in\right.$ $\Delta\}$ is an open cover of $T_{N} \operatorname{emb}(\Delta)$.

Proof. Check that

$$
T_{N} \operatorname{emb}(\Delta)-U_{\sigma}=\bigcup_{\tau \not \subset \sigma} \operatorname{orb} \tau
$$

is closed.

Proposition 2.15. If $\Delta$ is a fan on $N_{\mathbb{R}}$, then $X=T_{N} \operatorname{emb}(\Delta)$ is noetherian and quasicompact.

Proof. Let $\sigma \in \Delta$. Write $U_{\sigma}=\bigcup_{\tau \leq \sigma} \operatorname{orb} \tau$. Each $\operatorname{orb} \tau$ is isomorphic to a torus, hence is quasi-compact. So $U_{\sigma}$ is quasi-compact. Thus $X=\cup_{\sigma \in \Delta} U_{\sigma}$ is quasicompact. The proof that $X$ is noetherian is similar.

Lemma 2.16. For any $\sigma \in \Delta$, the value of the structure sheaf $\mathcal{O}$ on the open set $U_{\sigma}$ is the commutative ring $\Gamma\left(U_{\sigma}, \mathcal{O}\right)=k\left[\mathcal{S}_{\sigma}\right]$.

Proof. Since every quasi-local ring $P$ in $U_{\sigma}$ dominates some quasi-local ring $P^{\prime}$ in $V\left(k\left[\mathcal{S}_{\sigma}\right]\right)$, by (2.3),

$$
\mathcal{O}\left(U_{\sigma}\right)=\bigcap_{P \in U_{\sigma}} P=\bigcap_{P \in V\left(k\left[\mathcal{S}_{\sigma}\right]\right)} P=k\left[\mathcal{S}_{\sigma}\right] .
$$


Proposition 2.17. Let $\sigma$ be a strictly convex polyhedral cone in $N_{\mathbb{R}}$. Then

$$
\mathrm{H}^{0}\left(U_{\sigma}, \mathcal{O}^{*}\right) \cong k^{*} \oplus \mathbb{Z}^{r}
$$

where $r=n-\operatorname{dim}_{\mathbb{R}}\left(\operatorname{Supp}_{\mathbb{Q}}(\sigma)\right)$ and

$$
\mathrm{H}^{1}\left(U_{\sigma}, \mathcal{O}^{*}\right)=0 \text {. }
$$

Proof. By Lemma 2.16, $\mathrm{H}^{0}\left(U_{\sigma}, \mathcal{O}^{*}\right)$ is the group of units of the ring $k\left[\mathcal{S}_{\sigma}\right]$ which admits only trivial units [7, p. 129]. The group of invertible elements in $\mathcal{S}_{\sigma}$ is computed in Proposition 2.4.

Consider a 1-cocycle $x \in \check{\mathrm{H}}^{1}\left(\mathcal{U} / U_{\sigma}, \mathcal{O}^{*}\right)$ for some open cover $\mathcal{U}=\left\{U_{i}\right\}$. Let $V_{\sigma}$ denote $V\left(k\left[\mathcal{S}_{\sigma}\right]\right)$. For each $i$ set $V_{i}=U_{i} \cap V_{\sigma}$. Then $\mathcal{V}=\left\{V_{i}\right\}$ is an open cover for $V_{\sigma}$ and by Proposition 2.4, we can assume that upon restriction to $\check{\mathrm{H}}^{1}\left(\mathcal{V} / V_{\sigma}, \mathcal{O}^{*}\right)$, $x$ is a coboundary. But $\mathcal{O}\left(V_{i}\right)=\bigcap_{P \in V_{i}} P=\bigcap_{Q \in U_{i}} Q=\mathcal{O}\left(U_{i}\right)$ since each $Q \in U_{i}$ dominates some $P \in V_{i}$. Therefore $x$ is also a coboundary for $\mathcal{U}$.

Lemma 2.18. If $\sigma$ is a rational cone, then $U_{\sigma}$ is equal to $\operatorname{Spec} k\left[\mathcal{S}_{\sigma}\right]$.

Lemma 2.19. If $\tau$ and $\sigma$ are two cones in $\Delta$, then $U_{\tau} \cap U_{\sigma}=U_{\tau \cap \sigma}$.

Lemma 2.20. Given any $x \in T_{N} \operatorname{emb}(\Delta)$, the local ring $\mathcal{O}_{x}$ is the element of $L$ corresponding to $x$.

Proof. Since $x$ is in orb $\sigma$ for some $\sigma \in \Delta, U_{\sigma}$ is an open neighborhood of $x$ and $x$ is in the closed orbit of $U_{\sigma}$. The local ring $\mathcal{O}_{x}$ is the localization of $k\left[\mathcal{S}_{\sigma}\right]$ at a prime ideal.

The $k$-points of $V\left(k\left[\mathcal{S}_{\sigma}\right]\right)$ correspond to the maximal ideals of $k\left[\mathcal{S}_{\sigma}\right]$ with residue field $k$. Hence

$$
\operatorname{Spec}_{k} k\left[\mathcal{S}_{\sigma}\right]=\operatorname{Hom}\left(\mathcal{S}_{\sigma}, k\right)
$$

where the right hand side is the set of semigroup homomorphisms from $\mathcal{S}_{\sigma}$ to $(k, *)$ which map 0 to 1 . In this case, since $\mathcal{S}_{\sigma}$ generates $M$ as a group, there is an injective map

$$
T_{N}=\operatorname{Hom}_{\mathbb{Z}}\left(M, k^{*}\right) \hookrightarrow \operatorname{Hom}\left(\mathcal{S}_{\sigma}, k\right)=\operatorname{Spec}_{k} k\left[\mathfrak{S}_{\sigma}\right] .
$$

The group $\operatorname{Hom}_{\mathbb{Z}}\left(M, k^{*}\right)$ acts on the set $\operatorname{Hom}\left(\mathcal{S}_{\sigma}, k\right)$ by point-wise multiplication. If $g \in \operatorname{Hom}_{\mathbb{Z}}\left(M, k^{*}\right), x \in \operatorname{Hom}\left(\mathcal{S}_{\sigma}, k\right)$, and $m \in \mathcal{S}_{\sigma}$, then $(g x)(m)=g(m) x(m)$.

Theorem 2.21. The action by the algebraic group $T_{N}$ extends to the $k$-points of $T_{N} \operatorname{emb}(\Delta)$. The orbits are precisely $\{k$-rational points of $\operatorname{orb} \sigma \mid \sigma \in \Delta\}$.

Proof. This can be checked as in [13, p. 11].

A map of fans $\phi:\left(N^{\prime}, \Delta^{\prime}\right) \rightarrow(N, \Delta)$ is a homomorphism $\phi: N^{\prime} \rightarrow N$ whose scalar extension $\phi: N_{\mathbb{R}}^{\prime} \rightarrow N_{\mathbb{R}}$ satisfies the property that for each $\sigma^{\prime} \in \Delta^{\prime}$, there exists $\sigma \in \Delta$ such that $\phi\left(\sigma^{\prime}\right) \subseteq \sigma$. The next result implies that for a fixed $k, T_{()} \operatorname{emb}()$ is a functor.

Theorem 2.22. A map of fans $\phi:\left(N^{\prime}, \Delta^{\prime}\right) \rightarrow(N, \Delta)$ gives rise to a morphism of ringed spaces

$$
\phi_{*}: T_{N^{\prime}} \operatorname{emb}\left(\Delta^{\prime}\right) \rightarrow T_{N} \operatorname{emb}(\Delta),
$$

whose restriction to the open set $T_{N^{\prime}}$ coincides with the homomorphism of algebraic tori

$$
\phi \otimes 1: T_{N^{\prime}}=N^{\prime} \otimes_{\mathbb{Z}} k^{*} \rightarrow T_{N}=N \otimes_{\mathbb{Z}} k^{*}
$$



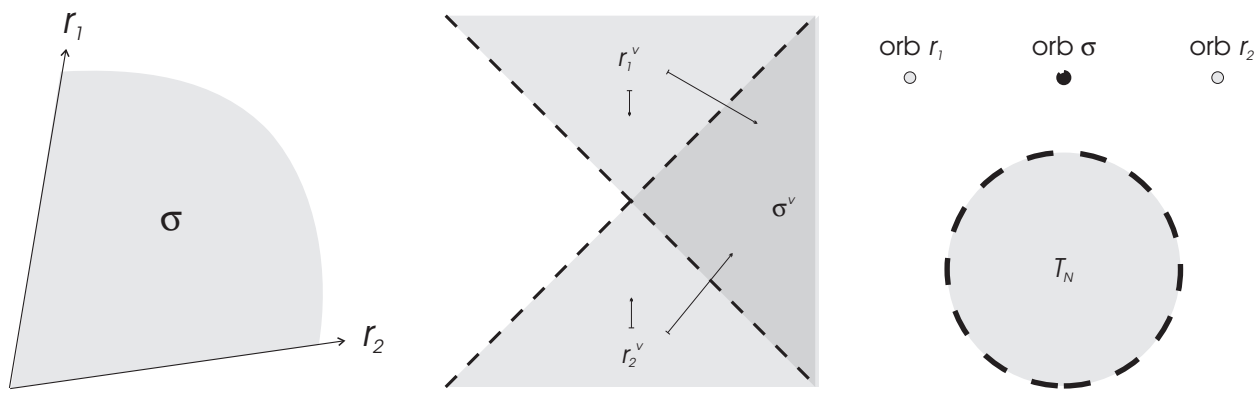

FIGURE 1. (a) the cone $\sigma \quad$ (b) the dual cones $\check{\sigma^{\prime}, r_{1}, r_{2}^{\check{r}}}$ (c) $U_{\sigma}$

arising from $\phi$. The morphism $\phi_{*}$ is equivariant with respect to the actions of $T_{N^{\prime}}$ and $T_{N}$ on the toric varieties.

Proof. The homomorphism $\phi: N^{\prime} \rightarrow N$ induces the homomorphism on dual modules $\phi^{*}: M \rightarrow M^{\prime}$. Tensoring with $k^{*}$ and $\mathbb{R}$ gives $\phi \otimes 1: T_{N^{\prime}} \rightarrow T_{N}$ and $\phi^{*}: M_{\mathbb{R}} \rightarrow M_{\mathbb{R}}^{\prime}$. Say $\phi\left(\sigma^{\prime}\right) \subseteq \sigma$ for $\sigma^{\prime} \in \Delta^{\prime}$ and $\sigma \in \Delta$. Then $\phi^{*}\left(\sigma^{\prime}\right) \subseteq\left(\sigma^{\prime}\right)^{\prime}$ and $\phi^{*}\left(\mathcal{S}_{\sigma}\right) \subseteq \mathcal{S}_{\sigma^{\prime}}$. So there is a homomorphism of $k$-algebras $\phi^{\#}: k\left[\mathcal{S}_{\sigma}\right] \rightarrow k\left[\mathcal{S}_{\sigma^{\prime}}\right]$ and a morphism of prime spectra Spec $k\left[\mathcal{S}_{\sigma^{\prime}}\right] \rightarrow \operatorname{Spec} k\left[\mathcal{S}_{\sigma}\right]$. Let $P^{\prime} \in T_{N^{\prime}} \operatorname{emb}\left(\Delta^{\prime}\right)$. From (2.2), we can pick $\sigma^{\prime} \in \Delta^{\prime}$ such that $P^{\prime} \in V\left(k\left[\mathcal{S}_{\sigma^{\prime}}\right]\right)$ and $\operatorname{dim} \sigma^{\prime}$ is minimal. Likewise pick $\sigma \in \Delta$ such that $\phi\left(\sigma^{\prime}\right) \subseteq \sigma$ and $\operatorname{dim} \sigma$ is minimal. Then there is a morphism $f_{\sigma^{\prime}}: V\left(k\left[\mathcal{S}_{\sigma^{\prime}}\right]\right) \rightarrow V\left(k\left[\mathcal{S}_{\sigma}\right]\right)$ and we set $\phi_{*}\left(P^{\prime}\right)$ equal to the point $f_{\sigma^{\prime}}\left(P^{\prime}\right)$. This defines the function $\phi_{*}: T_{N^{\prime}} \operatorname{emb}\left(\Delta^{\prime}\right) \rightarrow T_{N} \operatorname{emb}(\Delta)$. Note that under $\phi_{*}$ the orbit orb $\sigma^{\prime}$ is mapped continuously into the orbit orb $\sigma$. Hence the open set $U_{\sigma^{\prime}}$ is mapped into $U_{\sigma}$. Define the homomorphism on rings $\Gamma\left(U_{\sigma}, \mathcal{O}\right) \rightarrow \Gamma\left(U_{\sigma^{\prime}}, \mathcal{O}\right)$ to be $\phi^{\#}: k\left[\mathcal{S}_{\sigma}\right] \rightarrow k\left[\mathcal{S}_{\sigma^{\prime}}\right]$. These local morphisms extend to give a morphism $\phi$ of locally ringed spaces with the desired properties.

Example 2.23. Consider a two-dimensional strictly convex cone $\sigma$ in $N_{\mathbb{R}}$, where $N=\mathbb{Z}^{2}$, as shown in Figure 1a. Let $\sigma=r_{1}+r_{2}$ where each $r_{i}$ is an irrational onedimensional cone. The dual cones $\check{\sigma}, r_{1}, r_{2}$ are shown in Figure $1 \mathrm{~b}$. The dashed lines contain no lattice points. In this case adjoining the inverse of any element of $\mathcal{S}_{\sigma}$ generates the group $M$. So $\mathrm{Spec}_{k} k\left[\mathcal{S}_{\sigma}\right]$ consists of two $T_{N}$-orbits: the closed orbit orb $\sigma$ which is isomorphic to Spec $k$ and the open set isomorphic to the torus $T_{N}$. Consider the set $U_{\sigma}$ which is obtained by attaching orb $r_{1}$ and orb $r_{2}$ to $V\left(k\left[\mathcal{S}_{\sigma}\right]\right)$. Observe that orb $r_{i}=\operatorname{Spec}\left(k\left[\mathcal{S}_{r_{i}}\right] / I_{r_{i}}\right)$, where $I_{r_{i}}$ is the ideal in $k\left[\mathcal{S}_{r_{i}}\right]$ generated by all monomials that are not invertible. Since $r_{i}$ is irrational, $I_{r_{i}}$ is a maximal ideal in $k\left[\mathcal{S}_{r_{i}}\right]$. So orb $r_{i} \cong$ Spec $k$. Therefore $U_{\sigma}$ looks like $T_{N}$ with three points attached to its border, as shown in Figure 1c. Note that orb $r_{i}$ consists of only one point, but is not closed in $U_{\sigma}$. Topologically, closure $\left(\operatorname{orb} r_{i}\right)=\operatorname{orb} r_{i} \cup \operatorname{orb} \sigma$ has dimension one in $U_{\sigma}$.

2.4. The toroidal embedding associated to a real hyperplane arrangement. As a standard reference we attempt to follow [14].

For a hyperplane arrangement $\mathcal{A}$ of full rank in a finite dimensional vector space $V$ over $\mathbb{R}$, we define a toroidal embedding $T_{N} \operatorname{emb}(\mathcal{A})$, depending both on $\mathcal{A}$ and a basis for $V$. If the arrangement $\mathcal{A}$ is defined over $\mathbb{Q}$, then $T_{N} \operatorname{emb}(\mathcal{A})$ is a toric 
variety. As far as we know, the toroidal embedding has never been used to study hyperplane arrangements.

Let $V$ be a real vector space of dimension $l$ with basis $\left\{e_{1}, \ldots, e_{l}\right\}$. Let $\mathcal{A}$ be a hyperplane arrangement in $V$. That is,

$$
\mathcal{A}=\left\{H_{i} \mid i=1, \ldots, n \text { and } H_{i} \subseteq V \text { is an affine subspace of codimension } 1\right\} .
$$

For each $i$ choose a polynomial $\alpha_{i}$ of degree 1 in $\mathbb{R}\left[x_{1}, \ldots, x_{l}\right]$ such that $H_{i}$ is the set of zeros of $\alpha_{i}$ in $V$ (where it is understood that $\left\{x_{1}, \ldots, x_{l}\right\}$ is a dual basis in $V^{*}$ and $\mathbb{R}\left[x_{1}, \ldots, x_{l}\right]$ is the symmetric algebra of $\left.V^{*}\right)$. Then $\bigcup_{i=1}^{n} H_{i}$ is the zero set of $Q(\mathcal{A})=\prod_{i=1}^{n} \alpha_{i}$. Let $L=L(\mathcal{A})$ be the set of nonempty intersections of elements of $\mathcal{A}$ ordered by reverse inclusion

$$
X \leq Y \Leftrightarrow Y \subseteq X .
$$

If $X \in L(\mathcal{A})$, then $\operatorname{rank}(X)=\operatorname{codim}_{V}(X)$. Maximal elements of $L(\mathcal{A})$ have the same rank. The rank of $\mathcal{A}$ is the rank of a maximal element of $L(\mathcal{A})$. The cone of $\mathcal{A}$, denoted $c \mathcal{A}$, is an $(l+1)$-arrangement in $V \times \mathbb{R} e_{0}$ with defining polynomial $x_{0} Q^{h}(\mathcal{A})$. By $Q^{h}(\mathcal{A})$ we mean the homogenization of $Q(\mathcal{A})$ in $\mathbb{R}\left[x_{0}, x_{1}, \ldots, x_{l}\right]$ with respect to the new dual basis element $x_{0}$. The central arrangement $c \mathcal{A}$ induces a polyhedral subdivision of $V \times \mathbb{R}$ into cones. The maximal cones in this subdivision are the closures (in the metric topology) of the individual chambers of $V \times \mathbb{R}-$ $\bigcup_{H \in c \mathcal{A}} H$. Thus $c \mathcal{A}$ defines a fan on $V \times \mathbb{R}$ consisting of the maximal cones as well as all of their faces. Let $\Phi(\mathcal{A})$ denote this fan. We leave the proof of the following to the reader.

Lemma 2.24. Let $\mathcal{A}$ be a hyperplane arrangement in $V$ and $\Phi(\mathcal{A})$ the polyhedral subdivision of $V \times \mathbb{R}$ defined above.

(1) If $\mathcal{A}$ has rank $l$, then each maximal cone $\sigma \in \Phi(\mathcal{A})$ is a strictly convex polyhedral cone.

(2) If each defining polynomial $\alpha_{i}$ can be chosen to have rational coefficients, then each $\sigma \in \Phi(\mathcal{A})$ is a rational polyhedral cone.

(3) $\Phi(\mathcal{A})$ is a complete fan on $V \times \mathbb{R}$.

Let $N$ be the $\mathbb{Z}$-lattice in $V \times \mathbb{R}$ with basis $\left\{e_{0}, e_{1}, \ldots, e_{l}\right\}$. If $\mathcal{A}$ is a real arrangement of rank $l$, there is a toroidal embedding

$$
T_{N} \operatorname{emb}(\mathcal{A})=T_{N} \operatorname{emb}(\Phi(\mathcal{A}))
$$

associated to the fan $\Phi(\mathcal{A})$. If the hypotheses of Lemma 2.24.1 and 2.24.2 are satisfied, then $T_{N} \operatorname{emb}(\mathcal{A})$ is a normal, complete variety. In general the ringed space $T_{N} \operatorname{emb}(\mathcal{A})$ comes equipped with an action of the torus $T_{N}$. As the next example shows, the choice of basis for $V$ plays a critical role in the toroidal embedding associated to $\mathcal{A}$.

Example 2.25. Consider the two-arrangement $\mathcal{A}$ consisting of three lines defined by $Q(\mathcal{A})=x y(x+y-1)$, where $\mathbb{R}^{2}$ has the standard basis. The cone $c \mathcal{A}$ consists of four planes in $\mathbb{R}^{3}$ defined by $Q(c \mathcal{A})=x y z(x+y-z)$. Since each $H$ in $c \mathcal{A}$ is rational, $T_{N} \operatorname{emb}(\mathcal{A})$ is a toric variety. Now suppose we pick a different basis for $\mathbb{R}^{2}$. For example, take $\left\{(\sqrt{2}, 1)^{\top},(1, \sqrt{2})^{\top}\right\}$. With respect to the second basis, the equation for $\mathcal{A}$ becomes

$$
Q(\mathcal{A})=(\sqrt{2} x+y)(x+\sqrt{2} y)(x+y-(\sqrt{2}-1)),
$$


and the equation for $c \mathcal{A}$ becomes

$$
Q(c \mathcal{A})=z(\sqrt{2} x+y)(x+\sqrt{2} y)(x+y-z(\sqrt{2}-1)) .
$$

Three of the four hyperplanes in $c \mathcal{A}$ are irrational, so $T_{N} \operatorname{emb}(\mathcal{A})$ is not a variety.

The next result shows that every complete toroidal embedding is a proper birational image of $T_{N} \operatorname{emb}(\mathcal{A})$ for some arrangement $\mathcal{A}$.

Proposition 2.26. Let $N=\mathbb{Z}^{r}, r \geq 2$, and $\Delta$ any complete fan on $N_{\mathbb{R}}=\mathbb{R}^{r}$. Then there is an arrangement $\mathcal{A}$ in a vector space $V \cong \mathbb{R}^{r-1}$ such that the fan $\Phi(\mathcal{A})$ on $N_{\mathbb{R}}=V \times \mathbb{R}$ is a finite subdivision of $\Delta$. The morphism $T_{N} \operatorname{emb}(\mathcal{A}) \rightarrow X=T_{N} \operatorname{emb}(\Delta)$ is proper and birational.

Proof. For each cone $\tau \in \Delta(r-1)$, let $H_{\tau}=\mathbb{R} \tau$ be the subspace spanned by $\tau$. Then $\mathcal{B}=\left\{H_{\tau} \mid \tau \in \Delta(r-1)\right\}$ is a central hyperplane arrangement in $N_{\mathbb{R}}$. Distinguish any $H \in \mathcal{B}$. Pick a coordinate system for $H$ and extend it to $N_{\mathbb{R}}$. Dehomogenize with respect to $H$ and let $V$ denote the image of $N_{\mathbb{R}}-H$. Under this dehomogenization process, $\mathcal{B}-\{H\}$ is mapped to a hyperplane arrangement $\mathcal{A}$ in $V$. Also, $\mathcal{B}=c \mathcal{A}$ and $\Phi(\mathcal{A})$ is a finite subdivision of $\Delta$. The rest follows from [13, Corollary $1.17, \mathrm{p}$. 23]

Topics of Section 2.4, namely the fan and toroidal embedding associated to an arrangement of hyperplanes, will be revisited in Examples 3.4, 3.5, 4.18, and Propositions 3.9, 4.17.

\section{Divisor THEORY FOR A TOROIDAL EMBEDDING}

3.1. Weil divisors. In [11] U. Krause has shown that a divisor theory can exist for non-Krull monoids. Basic to his work is the notion of essential states. In our context, the rank one valuations on $k(M)$ associated to the one-dimensional cones $\rho \in \Delta(1)$ (Section 2.2) play the role of the essential states. In this section we show that a divisor theory exists for the toroidal embedding $X=T_{N} \operatorname{emb}(\Delta)$ defined in Section 2.3. The divisor class group $\mathrm{Cl}(X)$ is defined which generalizes the usual definition for Krull schemes.

Let $X=T_{N} \operatorname{emb}(\Delta)$ be the toroidal embedding associated to a fan $\Delta$. Let $X_{1}$ denote the points of $X$ with codimension one. Associated to each $x \in X_{1}$ is a rank one valuation $v_{x}$ on $k(M)$. Let $G_{x}$ denote the value group. Because $T_{N}$ is an open subset of $X$ and $X-T_{N}$ is a union of finitely many $T_{N}$-orbits, there are only finitely many $x \in X_{1}$ with non-discrete rank one valuations and by Lemma 2.8 these correspond to $\rho \in \Delta(1)$ which are irrational. For all $x \in X_{1}, G_{x}$ is a $\mathbb{Z}$-lattice. Define the group of divisors to be

$$
W=\coprod_{x \in X_{1}} G_{x} .
$$

Let $K^{*}=k(M)^{*}$ denote the group of units in the field of rational functions on $X$. The assignment $f \mapsto \sum_{x} v_{x}(f)$ defines a homomorphism of groups $K^{*} \rightarrow W$. The class group $\mathrm{Cl}(X)$ is defined to be the cokernel of this homomorphism.

$$
K^{*} \rightarrow \coprod_{x \in X_{1}} G_{x} \rightarrow \mathrm{Cl}(X) \rightarrow 0
$$

Comparing (3.1) to the counterpart for $T_{N}$, and using the fact that $\mathrm{Cl}\left(T_{N}\right)=0$, we see that $\mathrm{Cl}(X)$ is generated by the image of the group $\bigsqcup_{\rho \in \Delta(1)} G_{\rho}$. If a function 
$f \in K^{*}$ has trivial valuation at each $x \in T_{N}$, then $f$ is necessarily a monomial. So the sequence

$$
M \rightarrow \coprod_{\rho \in \Delta(1)} G_{\rho} \rightarrow \mathrm{Cl}(X) \rightarrow 0
$$

is exact.

Example 3.1. Let $\rho \in N_{\mathbb{R}}$ be a one-dimensional cone. Let $R=k\left[\mathcal{S}_{\rho}\right]$. The toroidal embedding $T_{N} \operatorname{emb}(\Delta(\rho))$ is the affine scheme $\operatorname{Spec} R$. We compute the class group of $R$ using (3.2). Since $\Delta(1)$ consists of only one cone, namely $\rho$, and the map $M \rightarrow G_{\rho}$ is surjective, it follows that $\mathrm{Cl}(R)=0$. Notice that the ring $R$ is not necessarily factorial by Proposition 2.2.

Proposition 3.2. Let $\sigma$ be an $n$-dimensional strictly convex polyhedral cone in $N_{\mathbb{R}}=\mathbb{R}^{n}$ and $X=U_{\sigma}=T_{N} \operatorname{emb}(\Delta(\sigma))$. Then $\mathrm{Cl}(X)$ is finite if and only if $\sigma$ is rational and simplicial.

Proof. Let $\Delta=\Delta(\sigma)$. If $\sigma$ is rational and simplicial, then $\mathrm{Cl}(X)$ is finite (for example [5, Lemma 3.4]). If $\mathrm{Cl}(X)$ is finite, then by (3.2), $\sum_{\rho \in \Delta(1)} \operatorname{rank}_{\mathbb{Z}}\left(G_{\rho}\right) \leq$ $\operatorname{rank}_{\mathbb{Z}} M=n$. Since $\sigma$ is $n$-dimensional, $\#(\Delta(1)) \geq n$. Therefore $\sigma$ is simplicial and $\operatorname{rank}_{\mathbb{Z}}\left(G_{\rho}\right)=1$ for each $\rho \in \Delta(1)$. By Lemma 2.8, $\rho$ is rational for each $\rho \in \Delta(1)$. Hence $\sigma$ is rational.

It will be shown in Example 3.7 that if the dimension of $\sigma$ is less than $n$, then the conclusion of Proposition 3.2 is false.

Example 3.3. Let $\sigma$ be a two-dimensional cone on $\mathbb{R}^{2}, \sigma=r_{0}+r_{1}$, where $r_{i}=$ $\mathbb{R}_{\geq 0} \eta_{i}$. Let $\Delta=\left\{\sigma, r_{0}, r_{1}, 0\right\}$ and $X=T_{N} \operatorname{emb}(\Delta)$. We compute $\mathrm{Cl}(X)$ using (3.2). If $r_{0}$ and $r_{1}$ are both rational, then $G_{r_{i}} \cong \mathbb{Z}$ and $\mathrm{Cl}(X)$ is finite. If $r_{0}$ is irrational, then $M \rightarrow G_{r_{0}}$ is an isomorphism so (3.2) splits and $G_{r_{1}} \cong \mathrm{Cl}(X)$. This shows that $\mathrm{Cl}(X)$ is finite if and only if $\sigma$ is rational. We illustrate the three possibilities with examples.

(1) Suppose $r_{0}$ and $r_{1}$ are both rational. For example, if $\eta_{0}=\left(\begin{array}{l}1 \\ 0\end{array}\right)$ and $\eta_{1}=\left(\begin{array}{l}1 \\ 2\end{array}\right)$, then $\mathrm{Cl}(X) \cong \mathbb{Z} / 2$.

(2) Suppose $r_{0}$ is rational and $r_{1}$ is irrational. For example, if $\eta_{0}=\left(\begin{array}{l}1 \\ 0\end{array}\right)$ and $\eta_{1}=\left(\begin{array}{c}1 \\ \sqrt{2}\end{array}\right)$, then $\mathrm{Cl}(X) \cong \mathbb{Z}$.

(3) Suppose $r_{0}$ and $r_{1}$ are both irrational. For example, if $\eta_{0}=\left(\begin{array}{c}1 \\ \sqrt{2}\end{array}\right)$ and $\eta_{1}=\left(\begin{array}{c}1 \\ 2 \sqrt{2}\end{array}\right)$, then $\mathrm{Cl}(X) \cong \mathbb{Z} \oplus \mathbb{Z}$

Example 3.4. Consider the following example from [14, p. 47]. Define two 2arrangements by the polynomials

$$
\begin{gathered}
Q(\mathcal{A})=x y(x-1)(x+1)(y-1)(y+1) \\
Q(\mathcal{B})=x y(x+y-1)(x+y+1)(x-y-1)(x-y+1)
\end{gathered}
$$

The arrangements have the same Poincaré polynomials. That is, $\pi(\mathcal{A}, t)=\pi(\mathcal{B}, t)=$ $(1+3 t)(1+3 t)$, hence the arrangements are $\pi$-equivalent. But they have distinct 
intersection posets $-L(\mathcal{A})$ is not equivalent to $L(\mathcal{B})$. Counting the number of one-dimensional faces shows that $\#(\Phi(\mathcal{A})(1))=22$ and $\#(\Phi(\mathcal{B})(1))=18$. Tensor (3.2) with $\mathbb{Q}$ to see that

$$
\begin{gathered}
\operatorname{dim}_{\mathbb{Q}} \mathrm{Cl}\left(T_{N} \operatorname{emb}(\mathcal{A})\right) \otimes \mathbb{Q}=19 \text { and } \\
\operatorname{dim}_{\mathbb{Q}} \mathrm{Cl}\left(T_{N} \operatorname{emb}(\mathcal{B})\right) \otimes \mathbb{Q}=15 .
\end{gathered}
$$

The varieties $T_{N} \operatorname{emb}(\mathcal{A})$ and $T_{N} \operatorname{emb}(\mathcal{B})$ are not isomorphic.

Example 3.5. Consider the following example from [14, p. 78]. Define two 2arrangements by the polynomials

$$
\begin{aligned}
& Q(\mathcal{A})=x(x-1) y(y-1)(2 y-2 x+1) \\
& Q(\mathcal{B})=(x-1)(x+1) y(x+y)(x-y)
\end{aligned}
$$

Consider the cones $c \mathcal{A}$ and $c \mathcal{B}$. The intersection posets $L(c \mathcal{A})$ and $L(c \mathcal{B})$ are not equivalent. The poset $L(c \mathcal{A})$ contains the two one-dimensional subspaces $z=0$, $x=0, x-z=0$ and $z=0, y=0, y-z=0$, both of which are subspaces of $z=0$. The poset $L(c \mathcal{B})$ has two triple lines, namely $y=0, y+x=0, y-x=0$ and $x-z=0, x+z=0, z=0$ which are not contained in the same hyperplane of $c \mathcal{B}$. In the notation of [14], L. Rose and H. Terao showed that $c \mathcal{A}$ and $c \mathcal{B}$ are $A$-equivalent, hence they are $\pi$-equivalent. The fan $\Phi(\mathcal{A})$ has exactly ten cones that are non-simplicial whereas the fan $\Phi(\mathcal{B})$ has twelve. The fan $\Phi(\mathcal{A})$ has two three-dimensional cones with five one-dimensional faces. The fan $\Phi(\mathcal{B})$ has no such three-dimensional cone. The local ring on $T_{N} \operatorname{emb}(\mathcal{A})$ at the corresponding closed orbit has class group $\mathbb{Z} \oplus \mathbb{Z}$. No such local ring exists for $T_{N} \operatorname{emb}(\mathcal{B})$. The varieties $T_{N} \operatorname{emb}(\mathcal{A})$ and $T_{N} \operatorname{emb}(\mathcal{B})$ are not isomorphic.

3.2. Cartier divisors. Let $\Delta$ be a fan on $N_{\mathbb{R}}$ and $X=T_{N} \operatorname{emb}(\Delta)$. Let $\Delta^{\prime}=\Delta(1) \cup$ $\Delta(0)$ be the fan made up of the cones in $\Delta$ of dimensions zero and one. Set $X^{\prime}=$ $T_{N} \operatorname{emb}\left(\Delta^{\prime}\right)$. Then $X^{\prime} \rightarrow X$ is an open immersion and $X^{\prime}$ contains each point of codimension one in $X$. Therefore $\mathrm{Cl}\left(X^{\prime}\right)=\mathrm{Cl}(X)$.

Theorem 3.6. In the above context, $\operatorname{Pic} X^{\prime}=\mathrm{Cl}(X)$.

Proof. According to Proposition 2.13, $X^{\prime}$ is a scheme and has an affine open covering $\mathcal{U}=\left\{V\left(k\left[\mathcal{S}_{\sigma}\right]\right) \mid \sigma \in \Delta(1)\right\}$. For each $\sigma \in \Delta(1)$, let $V_{\sigma}$ denote $V\left(k\left[\mathcal{S}_{\sigma}\right]\right)$. By Proposition 2.4, $\mathrm{H}^{1}\left(V_{\sigma}, \mathcal{O}^{*}\right)=\operatorname{Pic} V_{\sigma}=0$ for each $\sigma \in \Delta(1)$. By the spectral sequence comparing Čech cohomology and derived functor cohomology, the Čech cohomology group $\check{\mathrm{H}}^{1}\left(\mathcal{U} / X^{\prime}, \mathcal{O}^{*}\right)$ gives the Picard group $\operatorname{Pic} X^{\prime}=\mathrm{H}^{1}\left(X^{\prime}, \mathcal{O}^{*}\right)$. Let $\mathcal{K}^{*}$ denote the constant sheaf $U \mapsto K^{*}$ on $X^{\prime}$. Define the sheaf of Cartier divisors $\mathcal{C}$ on $X^{\prime}$ by the exact sequence

$$
1 \rightarrow \mathcal{O}^{*} \rightarrow \mathcal{K}^{*} \rightarrow \mathcal{C} \rightarrow 0
$$

Since $\mathcal{K}^{*}$ is a constant sheaf and $X^{\prime}$ is integral, $\mathrm{H}^{1}\left(X^{\prime}, \mathcal{K}^{*}\right)=0$ so $\mathrm{H}^{0}\left(X^{\prime}, \mathcal{C}\right) \rightarrow$ $\mathrm{H}^{1}\left(X^{\prime}, \mathcal{O}^{*}\right)$ is surjective. Since on $V_{\sigma}$ we have $\mathrm{H}^{1}\left(V_{\sigma}, \mathcal{O}^{*}\right)=0$, it follows that $\mathrm{H}^{0}\left(V_{\sigma}, \mathrm{C}\right)=K^{*} / \mathrm{H}^{0}\left(V_{\sigma}, \mathcal{O}^{*}\right)$. Define the sheaf of divisors $\mathcal{W}$ on $X^{\prime}$ by $U \mapsto \coprod_{x \in U_{1}} G_{x}$. Define a morphism of sheaves $\mathcal{C} \rightarrow \mathcal{W}$ by mapping a function $f \in K^{*}$ to $\sum v_{x}(f)$. On $V_{\sigma}$, Example 3.1 shows that $\mathrm{Cl}\left(V_{\sigma}\right)=0$. If a function $f \in K^{*}$ maps to 0 in $\mathrm{H}^{0}(U, \mathcal{W})$, then $f$ is a global unit on $U, f \in \mathrm{H}^{0}\left(U, \mathcal{O}^{*}\right)$. Therefore $\mathrm{H}^{0}\left(V_{\sigma}, \mathcal{C}\right) \cong$ $\mathrm{H}^{0}\left(V_{\sigma}, \mathcal{W}\right)$. But this implies global sections are also isomorphic, $\mathrm{H}^{0}\left(X^{\prime}, \mathcal{C}\right) \cong \mathrm{H}^{0}\left(X^{\prime}, \mathcal{W}\right)$. Because the groups of principal divisors agree for $\mathcal{C}$ and $\mathcal{W}$, the result follows. 
Example 3.7. As in Example 2.6, consider a two-dimensional cone $\sigma=r_{0}+r_{1}$ in $N_{\mathbb{R}}=\mathbb{R}^{3}$, where $r_{i}=\mathbb{R}_{\geq 0} \eta_{i}$. Suppose $\Delta=\left\{\sigma, r_{0}, r_{1}, 0\right\}, \Delta^{\prime}=\left\{r_{0}, r_{1}, 0\right\}$, $X=T_{N} \operatorname{emb}(\Delta), X^{\prime}=T_{N} \operatorname{emb}\left(\Delta^{\prime}\right)$. Then Theorem 3.6 says $\operatorname{Pic} X^{\prime}=\operatorname{Cl}(X)$. Compute $\operatorname{Pic} X^{\prime}=\mathrm{H}^{1}\left(X^{\prime}, \mathcal{O}^{*}\right)$ as the first Cech cohomology group $\check{\mathrm{H}}^{1}\left(\mathcal{U} / X^{\prime}, \mathcal{O}^{*}\right)$ of the open affine cover $\mathcal{U}=\left\{U_{r_{0}}, U_{r_{1}}\right\}$. The Čech complex of $\mathcal{U}$ is

$$
0 \rightarrow \mathrm{H}^{0}\left(U_{r_{0}}, \mathcal{O}^{*}\right) \oplus \mathrm{H}^{0}\left(U_{r_{1}}, \mathcal{O}^{*}\right) \stackrel{\delta^{0}}{\longrightarrow} \mathrm{H}^{0}\left(T_{N}, \mathcal{O}^{*}\right) \stackrel{\delta^{1}}{\longrightarrow} 0
$$

where $\mathrm{H}^{0}\left(U_{r_{i}}, \mathcal{O}^{*}\right) \cong k^{*} \oplus\left(\eta_{i}^{\perp} \cap M\right)$ and $\mathrm{H}^{0}\left(T_{N}, \mathcal{O}^{*}\right) \cong k^{*} \oplus M$, by Proposition 2.17. Also $\eta_{i}^{\perp} \cap M$ has rank equal to $3-\operatorname{dim}_{\mathbb{R}}\left(\operatorname{Supp}_{\mathbb{Q}}\left(\eta_{i}\right)\right)$. It follows from (3.4) that

$$
\mathrm{Cl}(X) \cong \operatorname{Pic} X^{\prime} \cong \frac{M}{\eta_{0}^{\perp} \cap M+\eta_{1}^{\perp} \cap M} .
$$

If $\eta_{0}$ and $\eta_{1}$ are both rational, then its easy to check that $\mathrm{Cl}(X)$ is finite. If both $\eta_{0}$ and $\eta_{1}$ are irrational, then $\eta_{0}^{\perp} \cap M+\eta_{1}^{\perp} \cap M$ has rank zero, one or two, so $\mathrm{Cl}(X)$ is infinite. If $\eta_{0}$ is rational, then we may assume $\eta_{0}$ is the first vector in a $\mathbb{Z}$-basis for $N$. Assume $\eta_{1}$ is irrational. Then $\eta_{1}^{\perp} \cap M$ has rank zero or one. It follows from (3.5) that the torsion-free part of $\mathrm{Cl}(X)$ has rank zero or one. The following examples illustrate the various possibilities.

(1) If $\eta_{0}=\left(\begin{array}{l}1 \\ 0 \\ 0\end{array}\right)$ and $\eta_{1}=\left(\begin{array}{l}\sqrt{2} \\ \sqrt{3} \\ \sqrt{5}\end{array}\right)$, then $\operatorname{Supp}_{\mathbb{Q}}\left(\eta_{1}\right)=N_{\mathbb{R}}$ so $\eta_{1}^{\perp} \cap M=0$ and $\mathrm{Cl}(X) \cong \mathbb{Z}$

(2) If $\eta_{0}=\left(\begin{array}{l}1 \\ 0 \\ 0\end{array}\right)$ and $\eta_{1}=\left(\begin{array}{c}\sqrt{2} \\ a \\ b\end{array}\right)$, where $a, b$ are rational numbers, then by Lemma 2.3, $\operatorname{Supp}_{\mathbb{Q}}\left(\eta_{1}\right)$ is spanned by the set $\left\{\left(\begin{array}{l}1 \\ 0 \\ 0\end{array}\right),\left(\begin{array}{l}0 \\ a \\ b\end{array}\right)\right\}$. Check that $\eta_{0}^{\perp} \cap M+$ $\eta_{1}^{\perp} \cap M$ is spanned by $\left\{\left(\begin{array}{l}0 \\ 1 \\ 0\end{array}\right),\left(\begin{array}{l}0 \\ 0 \\ 1\end{array}\right)\right\}$ so $\mathrm{Cl}(X) \cong \mathbb{Z}$.

(3) If $\eta_{0}=\left(\begin{array}{l}1 \\ 0 \\ 0\end{array}\right)$ and $\eta_{1}=\left(\begin{array}{c}a \\ \sqrt{2} \\ b\end{array}\right)$, where $a, b$ are integers, then by Lemma 2.3, $\operatorname{Supp}_{\mathbb{Q}}\left(\eta_{1}\right)$ is spanned by the set $\left\{\left(\begin{array}{l}0 \\ 1 \\ 0\end{array}\right),\left(\begin{array}{l}a \\ 0 \\ b\end{array}\right)\right\}$. Check that $\eta_{0}^{\perp} \cap M+\eta_{1}^{\perp} \cap M$ is spanned by $\left\{\left(\begin{array}{c}b \\ 0 \\ -a\end{array}\right),\left(\begin{array}{l}0 \\ 1 \\ 0\end{array}\right),\left(\begin{array}{l}0 \\ 0 \\ 1\end{array}\right)\right\}$ so $\mathrm{Cl}(X) \cong \mathbb{Z} /(b)$. This shows that Proposition 3.2 does not hold if the dimension of the cone $\sigma$ is less than the dimension of $N_{\mathbb{R}}$.

Proposition 3.8. Let $\Delta$ be a complete fan on $N_{\mathbb{R}}$. Assume for each cone $\sigma \in \Delta$ of dimension $n-1$, the one-dimensional subspace $\sigma^{\perp}$ of $M_{\mathbb{R}}$ is irrational. Then every Cartier divisor on $X=T_{N} \operatorname{emb}(\Delta)$ is principal, hence $\mathrm{H}^{1}\left(X, \mathcal{O}^{*}\right)=0$.

Proof. Choose a Cartier divisor $f \in \Gamma\left(X, \mathcal{K}^{*} / \mathcal{O}^{*}\right)$. Let $\Delta(n)=\left\{\sigma_{1}, \sigma_{2}, \ldots, \sigma_{m}\right\}$. For each $i$, set $U_{i}=U_{\sigma_{i}}=T_{N} \operatorname{emb}\left(\Delta\left(\sigma_{i}\right)\right)$. By Proposition 2.17, for each $i, \mathrm{H}^{1}\left(U_{i}, \mathcal{O}^{*}\right)=$ 
0 . Therefore $\left.f\right|_{U_{i}}$ is principal, hence $f$ is represented on the open cover $\left\{U_{i}\right\}_{i=1}^{m}$ by $\left\{\left(f_{i}, U_{i}\right)\right\}_{i=1}^{m}$ where $f_{i} \in K^{*}$ and for each pair $i, j, f_{i} / f_{j} \in \Gamma\left(U_{i} \cap U_{j}, \mathcal{O}^{*}\right)$. After scaling $f$ by a principal Cartier divisor, we may assume $f_{1}=1$. Since $\Delta$ is complete, there is some $\sigma_{i}, i \geq 2$, such that $\sigma_{1} \cap \sigma_{i} \in \Delta(n-1)$. Since $\left(\sigma_{1} \cap \sigma_{i}\right)^{\perp}$ is irrational, Proposition 2.17 gives $\Gamma\left(U_{i} \cap U_{j}, \mathcal{O}^{*}\right)=k^{*}$. So $f_{i} / f_{1}=f_{i} \in k^{*}$. By finite induction and since $\Delta$ is complete, we can show that $f_{j} \in k^{*}$ for each $j$. Therefore $f$ represents a global section of $\mathcal{O}^{*} / \mathcal{O}^{*}=1$. So $f$ is a principal Cartier divisor. Let $\mathrm{C}$ denote the quotient sheaf $\mathcal{K}^{*} / \mathcal{O}^{*}$ on $X$. Since $\mathcal{K}^{*}$ is constant and $X$ is irreducible, $\mathrm{H}^{1}\left(X, \mathcal{K}^{*}\right)=0$. The short exact sequence (3.3) gives rise to the exact sequence

$$
1 \rightarrow \Gamma\left(X, \mathcal{O}^{*}\right) \rightarrow \Gamma\left(X, \mathcal{K}^{*}\right) \rightarrow \Gamma(X, \mathcal{C}) \rightarrow \mathrm{H}^{1}\left(X, \mathcal{O}^{*}\right) \rightarrow 0
$$

which combines with the above argument to finish the proof.

Proposition 3.9. If $\mathcal{A}$ is a real hyperplane arrangement in $V=N_{\mathbb{R}}$ defined by $Q(\mathcal{A})=$ $\prod \alpha_{i}$ such that each $\alpha_{i}$ is irrational, then $\mathrm{H}^{1}\left(T_{N} \operatorname{emb}(\mathcal{A}), \mathcal{O}^{*}\right) \cong \mathbb{Z}$.

Proof. Decompose $\Phi=\Phi(\mathcal{A})$ into $\Phi_{-} \cup \Phi_{+}$where $\Phi_{+}$(resp. $\Phi_{-}$) consists of those cones $\sigma \in \Phi$ such that $x_{0}$ is non-negative (resp. non-positive) as a function on $\sigma$. So $\Phi_{0}=\Phi_{-} \cap \Phi_{+}$is a fan on the hyperplane $H_{0}$ where $x_{0}=0$. Let $X_{-}=$ $T_{N} \operatorname{emb}\left(\Phi_{-}\right), X_{+}=T_{N} \operatorname{emb}\left(\Phi_{+}\right), X_{0}=T_{N} \operatorname{emb}\left(\Phi_{0}\right)$. A slight modification of the proof of Proposition 3.8 shows that $\mathrm{H}^{1}\left(X_{-}, \mathcal{O}^{*}\right)=0$ and $\mathrm{H}^{1}\left(X_{+}, \mathcal{O}^{*}\right)=0$. The group of global units on $X_{i}$ is $k^{*} \times\left(\Phi_{i}\right)^{\perp} \cap M$. For $i=-$ and $i=+$, this group is $k^{*}$. But $\left(\Phi_{0}\right)^{\perp} \cap M \cong \mathbb{Z}$. The Mayer-Vietoris sequence corresponding to the double cover $X=X_{-} \cup X_{+}$is

$$
\begin{aligned}
\cdots \rightarrow \mathrm{H}^{i-1}\left(X_{0}, \mathcal{O}^{*}\right) \rightarrow \mathrm{H}^{i}\left(X, \mathcal{O}^{*}\right) \rightarrow \mathrm{H}^{i}\left(X_{-}, \mathcal{O}^{*}\right) \oplus \mathrm{H}^{i}\left(X_{+}, \mathcal{O}^{*}\right) & \\
& \rightarrow \mathrm{H}^{i}\left(X_{0}, \mathcal{O}^{*}\right) \rightarrow \cdots .
\end{aligned}
$$

In low dimensions this is

$$
1 \rightarrow k^{*} \rightarrow k^{*} \oplus k^{*} \rightarrow k^{*} \oplus \mathbb{Z} \rightarrow \mathrm{H}^{1}\left(X, \mathcal{O}^{*}\right) \rightarrow 0 .
$$

Therefore $\mathrm{H}^{1}\left(X, \mathcal{O}^{*}\right)=\mathbb{Z}$.

Example 3.10. We compute some Čech cohomology groups of $X=T_{N} \operatorname{emb}(\Delta)$ for a complete two-dimensional fan $\Delta$ on $\mathbb{R}^{2}$ which has exactly three maximal cones. Let $\Delta(2)=\left\{\sigma_{0}, \sigma_{1}, \sigma_{2}\right\}, \Delta(1)=\left\{r_{0}, r_{1}, r_{2}\right\}$. Suppose $\sigma_{i} \cap \sigma_{i+1}=r_{i}$ with subscripts interpreted $(\bmod 3)$. Then $T_{N} \operatorname{emb}(\Delta)$ has an open cover $\mathcal{U}=\left\{U_{\sigma_{0}}, U_{\sigma_{1}}, U_{\sigma_{2}}\right\}$ and $U_{\sigma_{i}} \cap U_{\sigma_{i+1}}=U_{r_{i}}$. Also $U_{0} \cap U_{1} \cap U_{2}=T_{N}$. We compute the cohomology groups $\check{\mathrm{H}}^{i}\left(\mathcal{U}, \mathcal{O}^{*}\right)$ with respect to the sheaf of units $\mathcal{O}^{*}$ on $T_{N} \operatorname{emb}(\Delta)$. We have $\Gamma\left(U_{\sigma_{i}}, \mathcal{O}^{*}\right)=k^{*}$ for each $i$. By Proposition $2.17 \mathrm{H}^{1}\left(U_{\sigma_{i}}, \mathcal{O}^{*}\right)=0$ for each $i$, so $\check{\mathrm{H}}^{1}\left(\mathcal{U} / X, \mathcal{O}^{*}\right)=\operatorname{Pic} X$. If $r_{i}$ is irrational, then $\mathcal{S}_{r_{i}}$ has no units, hence

$$
\Gamma\left(U_{r_{i}}, \mathcal{O}^{*}\right)= \begin{cases}k^{*} & \text { if } r_{i} \text { is irrational, } \\ k^{*} \oplus \mathbb{Z} & \text { if } r_{i} \text { is rational }\end{cases}
$$

for each $i$. Also $\Gamma\left(T_{N}, \mathcal{O}^{*}\right)=k^{*} \oplus \mathbb{Z}^{2}$. The Čech complex for $\mathcal{U}$ is

$$
0 \rightarrow \bigoplus_{i=0}^{2} \Gamma\left(U_{\sigma_{i}}, \mathcal{O}^{*}\right) \stackrel{\delta^{0}}{\longrightarrow} \bigoplus_{i=0}^{2} \Gamma\left(U_{r_{i}}, \mathcal{O}^{*}\right) \stackrel{\delta^{1}}{\longrightarrow} \Gamma\left(T_{N}, \mathcal{O}^{*}\right) \stackrel{\delta^{2}}{\longrightarrow} 0
$$


(1) Suppose each $r_{i}$ is irrational. Then (3.8) becomes

$$
0 \rightarrow k^{*} \oplus k^{*} \oplus k^{*} \stackrel{\delta^{0}}{\longrightarrow} k^{*} \oplus k^{*} \oplus k^{*} \stackrel{\delta^{1}}{\longrightarrow} k^{*} \oplus \mathbb{Z}^{2} \stackrel{\delta^{2}}{\longrightarrow} 0 .
$$

Therefore $\check{\mathrm{H}}^{0}\left(\mathcal{U}, \mathcal{O}^{*}\right)=k^{*}, \check{\mathrm{H}}^{1}\left(\mathcal{U}, \mathcal{O}^{*}\right)=0, \check{\mathrm{H}}^{2}\left(\mathcal{U}, \mathcal{O}^{*}\right)=\mathbb{Z}^{2}$.

(2) Suppose $r_{0}$ is rational, but $r_{1}$ and $r_{2}$ are irrational. Then (3.8) becomes

$$
0 \rightarrow k^{*} \oplus k^{*} \oplus k^{*} \stackrel{\delta^{0}}{\longrightarrow} k^{*} \oplus \mathbb{Z} \oplus k^{*} \oplus k^{*} \stackrel{\delta^{1}}{\longrightarrow} k^{*} \oplus \mathbb{Z}^{2} \stackrel{\delta^{2}}{\longrightarrow} 0 .
$$

Therefore $\check{\mathrm{H}}^{0}\left(\mathcal{U}, \mathcal{O}^{*}\right)=k^{*}, \check{\mathrm{H}}^{1}\left(\mathcal{U}, \mathcal{O}^{*}\right)=0, \check{\mathrm{H}}^{2}\left(\mathcal{U}, \mathcal{O}^{*}\right)=\mathbb{Z}$.

(3) Suppose $r_{0}$ and $r_{1}$ are both rational, but $r_{2}$ is irrational. Then (3.8) becomes

$$
0 \rightarrow k^{*} \oplus k^{*} \oplus k^{*} \stackrel{\delta^{0}}{\longrightarrow} k^{*} \oplus \mathbb{Z} \oplus k^{*} \oplus \mathbb{Z} \oplus k^{*} \stackrel{\delta^{1}}{\longrightarrow} k^{*} \oplus \mathbb{Z}^{2} \stackrel{\delta^{2}}{\longrightarrow} 0 .
$$

Therefore $\check{\mathrm{H}}^{0}\left(\mathcal{U}, \mathcal{O}^{*}\right)=k^{*}, \check{\mathrm{H}}^{1}\left(\mathcal{U}, \mathcal{O}^{*}\right)=0, \check{\mathrm{H}}^{2}\left(\mathcal{U}, \mathcal{O}^{*}\right)=\mathbb{Z} / b$, where $b$ is the index in $M$ of the subgroup spanned by $M \cap\left(r_{0}^{\perp} \cup r_{1}^{\perp}\right)$. Does the 2-cocycle of order $b$ correspond to an element of order $b$ in the Brauer group of $T_{N} \operatorname{emb}(\Delta)$ ? If yes, can the methods of [4] be used to construct the Azumaya algebra?

3.3. Homogeneous coordinate ring. Let $\Delta$ be a fan on $N_{\mathbb{R}}$ such that $\Delta(1)$ spans $N_{\mathbb{R}}$. Fix $\sigma \in \Delta$ a cone with dimension at least one. Then $k\left[\mathcal{S}_{\sigma}\right]=\bigcap_{\rho \in \sigma(1)} k\left[\mathcal{S}_{\rho}\right]$ so of course the rings $k\left[\mathcal{S}_{\rho}\right]$ play a fundamental role in the theory of toroidal embeddings. This was pointed out by D. Cox in [1]. If $\Delta$ is rational, the homogeneous coordinate ring of $X=T_{N} \operatorname{emb}(\Delta)$ is a polynomial ring $S=k\left[x_{\rho} \mid \rho \in \Delta(1)\right]$. If $\rho \in$ $\Delta(1)$ is rational, then the valuation $v_{\rho}$ is discrete with value group $G_{\rho} \cong \mathbb{Z}$ and the ring $k\left[\mathcal{S}_{\rho}\right]$ is isomorphic to $k\left[x_{1}\right]\left[x_{2}, x_{2}^{-1}, \ldots, x_{n}, x_{n}^{-1}\right]$. The component $k\left[x_{1}\right]$ can be viewed as the semigroup ring $k\left[G_{\rho}^{+}\right]$and the component $k\left[x_{2}, x_{2}^{-1}, \ldots, x_{n}, x_{n}^{-1}\right]$ as the group ring $k\left[\rho^{\perp} \cap M\right]$. If $\rho$ is irrational, then $k\left[\mathcal{S}_{\rho}\right]$ is isomorphic to $k\left[G_{\rho}^{+}\right]\left[\rho^{\perp} \cap M\right]$. Note that because $\rho$ is irrational, the ring $k\left[G_{\rho}^{+}\right]$is non-noetherian and is not a polynomial ring. It is useful to use multiplicative notation and write $k\left[G_{\rho}^{+}\right]=$ $k\left[x_{\rho}^{\alpha} \mid \alpha \in G_{\rho}^{+}\right]$where $x_{\rho}$ is an indeterminate.

For the fan $\Delta$ with toroidal embedding $X=T_{N} \operatorname{emb}(\Delta)$ we define the homogeneous coordinate ring to be the semigroup ring

$$
S=\bigotimes_{\rho \in \Delta(1)} k\left[G_{\rho}^{+}\right]=k\left[\coprod_{\rho \in \Delta(1)} G_{\rho}^{+}\right] .
$$

For a rational fan, this agrees with the definition given in [1]. If $\sigma$ is as above, set $x^{\hat{\sigma}}=\prod_{\rho \notin \sigma(1)} x_{\rho}$. Then $x^{\hat{\sigma}}$ is a monomial in $S$. Let $S_{\sigma}$ be the localization of $S$ at $x^{\hat{\sigma}}, S_{\sigma}=S\left[\prod_{\rho \notin \sigma(1)} x_{\rho}^{-1}\right]$. By $\mathrm{Cl}(X)^{+}$we denote the monoid of divisor classes in the image of the monoid $\coprod_{\rho \in \Delta(1)} G_{\rho}^{+}$. The ring $S$ has a grading by $\operatorname{Cl}(X)^{+}$. A monomial $\alpha=\prod x_{\rho}^{\alpha_{\rho}}$ corresponds to an effective divisor $\sum \alpha_{\rho}$ in $\amalg G_{\rho}^{+}$and we define the degree of $\alpha$ to be the class of this divisor in $\mathrm{Cl}(X)$. There is a grading on $S_{\sigma}$ by the monoid image of $\left(\coprod_{\rho \in \sigma(1)} G_{\rho}^{+}\right) \oplus\left(\coprod_{\rho \notin \sigma(1)} G_{\rho}\right)$ in $\mathrm{Cl}(X)$. The degree zero part of $S_{\sigma}$ is generated by those monomials $\alpha \in S$ such that $\alpha=\prod x_{\rho}^{\alpha_{\rho}}, \alpha_{\rho} \geq 0$ for all $\rho \in \sigma(1)$ and the divisor $\sum \alpha_{\rho}$ in $\amalg G_{\rho}$ is in the image of $M \rightarrow \amalg G_{\rho}$. In this case, we see that the homomorphism [1, Lemma 2.2]

$$
\phi_{\sigma}: k\left[\mathcal{S}_{\sigma}\right] \rightarrow\left(S_{\sigma}\right)_{0}
$$

which maps a monomial $\chi^{m}$ to $\prod x_{\rho}^{\left\langle\eta_{\rho}, m\right\rangle}$ is an isomorphism. 


\section{A SCHEME THAT DOES NOT DEPEND ON $N$}

It is possible to define a scheme $X(\Delta)$ associated to $\Delta$ that does not depend on the $\mathbb{Z}$-lattice $N$. Some of the functorial properties of $X(\Delta)$ are derived. The Picard group and the Brauer group of $X(\Delta)$ are studied.

4.1. The Definition of $X(\Delta)$. Let $\sigma$ be a strictly convex polyhedral cone in $N_{\mathbb{R}}$. First let us consider the case where the cone is zero-dimensional. Then the dual cone is $M_{\mathbb{R}}$ which is an abelian group. The group algebra is $k\left[M_{\mathbb{R}}\right]$. Since $M_{\mathbb{R}}$ has infinite torsion-free rank, the Krull dimension of $k\left[M_{\mathbb{R}}\right]$ is infinite [10, p. 80]. The group algebra $k\left[M_{\mathbb{R}}\right]$ admits only trivial units [7, p. 129], hence the group of units of $k\left[M_{\mathbb{R}}\right]$ is isomorphic to $k^{*} \times M_{\mathbb{R}}$. Denote by $\operatorname{Spec}_{k}$ the points in Spec corresponding to points with residue field $k$.

Proposition 4.1. The set $\mathrm{Spec}_{\mathbb{C}} \mathbb{C}\left[M_{\mathbb{R}}\right]$ is a group and the natural map

$$
\eta: \operatorname{Spec}_{\mathbb{C}} \mathbb{C}\left[M_{\mathbb{R}}\right] \rightarrow \operatorname{Spec}_{\mathbb{C}} \mathbb{C}[M]
$$

is a surjective homomorphism of groups.

Proof. It is enough to prove this for the one-dimensional case where $N=\mathbb{Z}$. Now $\operatorname{Spec}_{\mathbb{C}} \mathbb{C}[\mathbb{R}]$ can be identified in the usual way with the group of $\mathbb{Z}$-module homomorphisms $\operatorname{Hom}_{\mathbb{Z}}\left(\mathbb{R}, \mathbb{C}^{*}\right)$. The inclusion $\mathbb{C}\left[x, x^{-1}\right] \rightarrow \mathbb{C}\left[x^{t} \mid t \in \mathbb{R}\right]$ induces a natural map

$$
\operatorname{Hom}_{\mathbb{Z}}\left(\mathbb{R}, \mathbb{C}^{*}\right) \rightarrow \operatorname{Hom}_{\mathbb{Z}}\left(\mathbb{Z}, \mathbb{C}^{*}\right) \cong \mathbb{C}^{*} .
$$

Under this map, a homomorphism $f: \mathbb{R} \rightarrow \mathbb{C}^{*}$ is mapped to $\left.f\right|_{\mathbb{Z}}: \mathbb{Z} \rightarrow \mathbb{C}^{*}$ hence (4.1) is a homomorphism of groups. This map has a section, obtained by modifying the usual exponential map $e^{z}: \mathbb{C} \rightarrow \mathbb{C}^{*}$. Given $\alpha \in \mathbb{C}^{*}$, write $\alpha=|\alpha| e^{i \theta}$ where $0 \leq \theta<2 \pi$, and define $f: \mathbb{R} \rightarrow \mathbb{C}^{*}$ by $f(t)=\alpha^{t}=|\alpha|^{t} e^{i \theta t}$. Therefore (4.1) is onto and splits.

Proposition 4.2. Let $\sigma$ be a convex polyhedral cone, and let $u$ be in $\sigma$. Then $\tau=\sigma \cap u^{\perp}$ is a convex polyhedral cone. All faces of $\sigma$ have this form, and

$$
\check{\tau}=\check{\sigma}+\mathbb{Z}_{\geq 0} \cdot(-u) \text {. }
$$

Proof. See [6, Prop. 2, p. 13]. If $\tau$ is a face of $\sigma$, then $\tau=\sigma \cap u^{\perp}$ for any $u$ in the relative interior of $\sigma \curvearrowleft \tau^{\perp}$. Given $y \in \tau, y+m u$ is in $\check{\sigma}$ for $m \gg 0, m \in \mathbb{Z}$.

Proposition 4.3. If $\sigma_{1}$ and $\sigma_{2}$ are convex polyhedral cones and $\tau=\sigma_{1} \cap \sigma_{2}$ is a face of both $\sigma_{1}$ and $\sigma_{2}$, then

$$
\check{\tau}=\sigma_{1}^{\check{ }}+\sigma_{2}^{\check{2}} .
$$

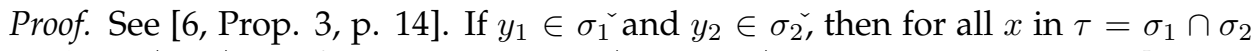
we have $\left\langle y_{i}, x\right\rangle \geq 0$ for $i=1,2$. Hence $\left\langle y_{1}+y_{2}, x\right\rangle \geq 0$ so $y_{1}+y_{2} \in \tau$. By [6, (12), p. 13] there exists $u \in \sigma_{1} \cap\left(-\sigma_{2}\right)^{\swarrow}$ with $\tau=\sigma \cap u^{\perp}=\sigma_{2} \cap u^{\perp}$. By Proposition 4.2,

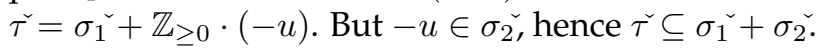

Let $\Delta$ be a fan of strictly convex polyhedral cones in $N_{\mathbb{R}}$. For each $\sigma \in \Delta$, let

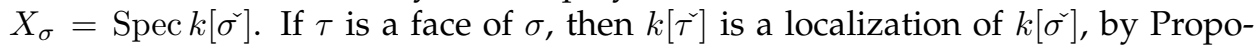
sition 4.2. If $\sigma_{1}$ and $\sigma_{2}$ have the common face $\tau=\sigma_{1} \cap \sigma_{2}$, then Proposition 4.3 shows that the natural map $k\left[\sigma_{1}^{\sim}\right] \otimes k\left[\sigma_{2}^{\sim}\right] \rightarrow k\left[\tau^{\sim}\right]$ is surjective. Therefore we can glue the $X_{\sigma}$ together to get a separated scheme

$$
X(\Delta)=\underset{\sigma \in \Delta}{\cup} X_{\sigma}
$$


The affine group scheme $\operatorname{Spec}_{k} k\left[M_{\mathbb{R}}\right]$ is a subset of $\operatorname{Spec}_{k} k[\sigma]$ for each $\sigma \in \Delta$ and acts as a group via the usual set inclusion:

$$
\operatorname{Spec}_{k} k\left[M_{\mathbb{R}}\right]=\operatorname{Hom}\left(M_{\mathbb{R}}, k^{*}\right) \subseteq \operatorname{Hom}\left(\check{\sigma}, k^{*}\right)=\operatorname{Spec}_{k} k[\check{\sigma}] .
$$

Theorem 4.4. The action by the group scheme $\operatorname{Spec}_{k} k\left[M_{\mathbb{R}}\right]$ extends to the k-points of $X(\Delta)$. The orbits are in one-to-one correspondence with the cones $\sigma \in \Delta$. The orbit space, with the topology inherited from $X(\Delta)$, is homeomorphic to $\mathfrak{T}(\Delta)$. Here $\mathfrak{T}$ is the functor defined in (1.1).

Proof. This can be checked as in [13, p. 11].

Theorem 4.5. For a field $k$ and $\mathbb{Z}$-lattice $N$, let $\Delta$ be a fan on $N_{\mathbb{R}}$. Then there is a morphism of ringed spaces

$$
\phi: X(\Delta) \rightarrow T_{N} \operatorname{emb}(\Delta)
$$

that is equivariant with respect to the actions of the groups $\operatorname{Spec}_{k} k\left[M_{\mathbb{R}}\right]$ and $T_{N}$. For $k=\mathbb{C}$, the morphism $\phi$ is surjective.

Proof. Let $P \in X(\Delta)$. From (4.2) we can pick $\sigma \in \Delta$ such that $P \in X_{\sigma}$ and $\operatorname{dim} \sigma$ is minimal. There is a $k$-algebra homomorphism $\phi^{\#}: k\left[\mathcal{S}_{\sigma}\right] \rightarrow k\left[\sigma^{\circ}\right]$ and a morphism

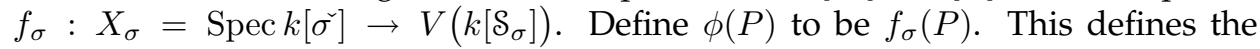
function $\phi$ on $X(\Delta)$. Note that $\phi$ maps $X_{\sigma}$ into $U_{\sigma}$. The morphism of sheaves $\phi^{\#}$

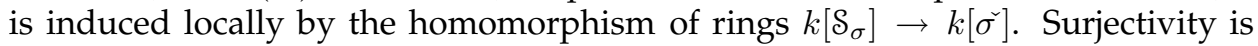
proved by applying Proposition 4.1 to the map $\phi$ restricted to orbits. The rest is left to the reader.

\subsection{The Picard group of $X(\Delta)$.}

Lemma 4.6. Let $\Delta$ be a rational fan on $N_{\mathbb{R}}$ and $U$ the open cover $\left\{X_{\sigma} \mid \sigma \in \Delta\right\}$ of $X(\Delta)$. Let $X=T_{N} \operatorname{emb}(\Delta)$ denote the toric variety associated to $\Delta$. Then for $p \geq 1$,

$$
\check{\mathrm{H}}^{p}\left(\mathcal{U} / X(\Delta), \mathcal{O}^{*}\right) \cong \check{\mathrm{H}}^{p}\left(X, \mathcal{O}^{*}\right) \otimes \mathbb{R} \cong \mathrm{H}^{p}\left(X, \mathcal{O}^{*}\right) \otimes \mathbb{R} .
$$

Proof. The algebra $k\left[\sigma^{\circ}\right]$ admits only trivial units, hence the group of units is $\mathrm{H}^{0}\left(X_{\sigma}, \mathcal{O}^{*}\right)=$

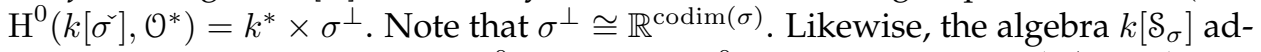
mits only trivial units, hence $\mathrm{H}^{0}\left(U_{\sigma}, \mathcal{O}^{*}\right)=\mathrm{H}^{0}\left(k\left[\mathcal{S}_{\sigma}\right], \mathcal{O}^{*}\right)=k^{*} \times\left(\sigma^{\perp} \cap M\right)$ and $\sigma^{\perp} \cap M \cong \mathbb{Z}^{\operatorname{codim}(\sigma)}$. Therefore $\mathrm{H}^{0}\left(X_{\sigma}, \mathcal{O}^{*}\right) / k^{*}=\mathrm{H}^{0}\left(U_{\sigma}, \mathcal{O}^{*}\right) / k^{*} \otimes \mathbb{R}$. Let $\mathcal{V}$ denote the open cover $\left\{U_{\sigma} \mid \sigma \in \Delta\right\}$ of $X$. Then $\check{\mathrm{H}}^{p}\left(\mathcal{V} / X, \mathcal{O}^{*}\right)=\check{\mathrm{H}}^{p}\left(X, \mathcal{O}^{*}\right)=\mathrm{H}^{p}\left(X, \mathcal{O}^{*}\right)$ by [2, Lemma 5]. Tensoring the Cech complex for the cover $\mathcal{V}$ and the sheaf $\mathcal{O}^{*}$ on $X$ gives the Cech complex for the cover $\mathcal{U}$ and the sheaf $\mathcal{O}^{*}$ on $X(\Delta)$. This proves the lemma.

Corollary 4.7. Let $\Delta$ be a rational fan on $N_{\mathbb{R}}$ and $U$ the open cover $\left\{X_{\sigma} \mid \sigma \in \Delta\right\}$ of $X(\Delta)$. Let $X=T_{N} \operatorname{emb}(\Delta)$ denote the toric variety associated to $\Delta$ and $K$ the function field of $X$. Then

$$
\check{\mathrm{H}}^{1}\left(\mathcal{U} / X(\Delta), \mathcal{O}^{*}\right)=\operatorname{Pic}(X) \otimes \mathbb{R}
$$

and

$$
\check{\mathrm{H}}^{2}\left(\mathcal{U} / X(\Delta), \mathcal{O}^{*}\right)=\mathrm{H}^{2}\left(K / X_{\text {ét }}, \mathbb{G}_{m}\right) \otimes \mathbb{R} .
$$

Proof. This follows directly from Lemma 4.6 and [2, Theorem 1]. 
Example 4.8. As in Example 3.10, we compute some Čech cohomology groups of $X(\Delta)$ for a complete two-dimensional fan $\Delta$ on $\mathbb{R}^{2}$ which has exactly three maximal cones. Let $\Delta(2)=\left\{\sigma_{0}, \sigma_{1}, \sigma_{2}\right\}, \Delta(1)=\left\{r_{0}, r_{1}, r_{2}\right\}$. Suppose $\sigma_{i} \cap \sigma_{i+1}=r_{i}$ with subscripts interpreted $(\bmod 3)$. Then $X(\Delta)$ has an open cover $\mathcal{U}=\left\{X_{\sigma_{0}}, X_{\sigma_{1}}, X_{\sigma_{2}}\right\}$ and $X_{\sigma_{i}} \cap X_{\sigma_{i+1}}=X_{r_{i}}$. Also $X_{0} \cap X_{1} \cap X_{2}=\operatorname{Spec} k\left[M_{\mathbb{R}}\right]$. We compute the cohomology groups $\check{\mathrm{H}}^{i}\left(\mathcal{U}, \mathcal{O}^{*}\right)$ with respect to the sheaf of units $\mathcal{O}^{*}$ on $X(\Delta)$. It follows from Proposition 4.9 below that $\check{\mathrm{H}}^{1}\left(\mathcal{U}, \mathcal{O}^{*}\right) \cong \mathrm{H}^{1}\left(X(\Delta), \mathcal{O}^{*}\right)=\operatorname{Pic} X(\Delta)$. We have $\Gamma\left(X_{\sigma_{i}}, \mathcal{O}^{*}\right)=k^{*}$ for each $i$. Since $\operatorname{dim} r_{i}=1$,

$$
\Gamma\left(X_{r_{i}}, \mathcal{O}^{*}\right)=k^{*} \times r_{i}^{\perp} \cong k^{*} \times \mathbb{R}
$$

for each $i$. Also $\Gamma\left(k\left[M_{\mathbb{R}}\right], \mathcal{O}^{*}\right) \cong k^{*} \times \mathbb{R}^{2}$. The Čech complex for $\mathcal{U}$ is

$$
0 \rightarrow \bigoplus_{i=0}^{2} \Gamma\left(X_{\sigma_{i}}, \mathcal{O}^{*}\right) \stackrel{\delta^{0}}{\longrightarrow} \bigoplus_{i=0}^{2} \Gamma\left(X_{r_{i}}, \mathcal{O}^{*}\right) \stackrel{\delta^{1}}{\longrightarrow} \Gamma\left(k\left[M_{\mathbb{R}}\right], \mathcal{O}^{*}\right) \stackrel{\delta^{2}}{\longrightarrow} 0
$$

which becomes, after substitution,

$$
0 \rightarrow k^{*} \oplus k^{*} \oplus k^{*} \stackrel{\delta^{0}}{\longrightarrow} k^{*} \times \mathbb{R} \oplus k^{*} \times \mathbb{R} \oplus k^{*} \times \mathbb{R} \stackrel{\delta^{1}}{\longrightarrow} k^{*} \times \mathbb{R}^{2} \stackrel{\delta^{2}}{\longrightarrow} 0 .
$$

Therefore $\check{\mathrm{H}}^{0}\left(\mathcal{U}, \mathcal{O}^{*}\right)=k^{*}, \check{\mathrm{H}}^{1}\left(\mathcal{U}, \mathcal{O}^{*}\right)=\mathbb{R}, \check{\mathrm{H}}^{2}\left(\mathcal{U}, \mathcal{O}^{*}\right)=0$.

Let $\sigma$ be a strictly convex polyhedral cone in $N_{\mathbb{R}}$ and $\sigma$ the dual cone in $M_{\mathbb{R}}$. We now show that the $k$-algebra $k\left[\sigma^{\complement}\right]$ is a union of finitely generated sub-k-algebras each of which is the coordinate ring of an affine toric variety. The lattice of these subalgebras is not a limit system in the category of toric varieties since the torus varies.

Let $\Omega$ be a finitely generated semigroup in $\sigma$ and $\bar{M}$ the subgroup of $M_{\mathbb{R}}$ spanned by $\Omega$. Assume $\Omega$ is integrally closed in $\bar{M}$. Let $S$ denote the semigroup ring $k[\Omega]$, which we view as a subring of the torus $k[\bar{M}]$. The Krull dimension of $S$ is finite and is equal to the rank of the torsion free abelian group $\bar{M}$. Set $\bar{N}=\operatorname{Hom}_{\mathbb{Z}}(\bar{M}, \mathbb{Z})$ and $\bar{N}_{\mathbb{R}}=\bar{N} \otimes \mathbb{R}$. Set $\bar{\sigma}=\left\{x \in \bar{N}_{\mathbb{R}} \mid\langle x, \omega\rangle \geq 0\right.$ for all $\left.\omega \in \Omega\right\}$. So $\bar{\sigma}$ is a strictly convex rational polyhedral cone in $\bar{N}_{\mathbb{R}}$ and $\Omega=\bar{\sigma} \cap \bar{M}$. Therefore Spec $k[\Omega]$ is isomorphic to the affine toric variety $T_{\bar{N}} \operatorname{emb}(\Delta(\bar{\sigma}))$.

In the above notation, let $\overline{M_{0}}$ denote the maximal subgroup of $\Omega$. If $\xi$ is a lattice point in the relative interior of $\bar{\sigma}$, then $\langle\xi, \omega\rangle$ is a non-negative integer for each $\omega \in \Omega$, and $\langle\xi, \omega\rangle=0$ if and only if $\omega$ is in $\overline{M_{0}}$. So taking inner products with $\xi$ turns $S$ into a graded $k$-algebra and the subring of elements of degree zero is the torus $k\left[\overline{M_{0}}\right]$.

Proposition 4.9. Let $\sigma$ be a strictly convex polyhedral cone in $N_{\mathbb{R}}$ and $\sigma$ the dual cone in

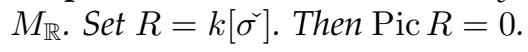

Proof. Let $P$ be an invertible $R$-module. Then $P$ is locally free of rank one and of finite presentation. So there is a finitely generated subsemigroup $\Omega$ of $\sigma$ such that if $S=k[\Omega]$, then there is an invertible $S$-module $P^{\prime}$ and $P \cong P^{\prime} \otimes_{S} R$. Furthermore we can pick $S$ so that $S$ is a normal domain which is the affine coordinate ring of an affine toric variety. It is well known that for such a $\operatorname{ring} S, \operatorname{Pic} S=0$. Hence, $P^{\prime}$ and $P$ are both free.

Let $\Delta$ be a fan on $N_{\mathbb{R}}$. Let $\Delta(n)=\left\{\sigma_{1}, \ldots, \sigma_{m}\right\}$. Since Pic $X_{\sigma}=0$ for each $\sigma \in \Delta$, every Cartier divisor on $X(\Delta)$ is split by the open cover $\mathcal{U}=\left\{X_{\sigma_{i}}\right\}_{i=1}^{m}$ 
and $\check{\mathrm{H}}^{1}\left(\mathcal{U}, \mathcal{O}^{*}\right) \cong \mathrm{H}^{1}\left(X(\Delta), \mathcal{O}^{*}\right)$. Given a Cartier divisor $f=\left\{\left(X_{\sigma_{i}}, f_{i}\right)\right\}_{i=1}^{m}$, after scaling we assume $f_{1}=1$. So for each $i>1$ we have

$$
f_{i}=f_{i} / f_{1} \in \Gamma\left(X_{\sigma_{1 i}}, \mathcal{O}^{*}\right) \subseteq k\left[M_{\mathbb{R}}\right]^{*} .
$$

So we can assume $f_{i} \in k\left[M_{\mathbb{R}}\right]^{*}$. That is, $f_{i}$ is a linear functional on $\left|\sigma_{i}\right|$ and for each pair $i, j$, the functions $\left.f_{i}\right|_{\sigma_{i j}}$ and $\left.f_{j}\right|_{\sigma_{i j}}$ are equal as linear functionals on $\left|\sigma_{i j}\right|$. Let $\mathrm{SF}(\Delta)$ denote the group of (real-valued) $\Delta$-linear support functions. Let $\mathcal{C}$ denote the sheaf of Cartier divisors on $X(\Delta)$. The mapping $\mathrm{SF}(\Delta) \rightarrow \Gamma(X(\Delta), \mathrm{C})$ defined by $f \mapsto\left\{\left(X_{\sigma_{i}},\left.f\right|_{\left|\sigma_{i}\right|}\right)\right\}_{i=1}^{m}$ induces a commutative diagram

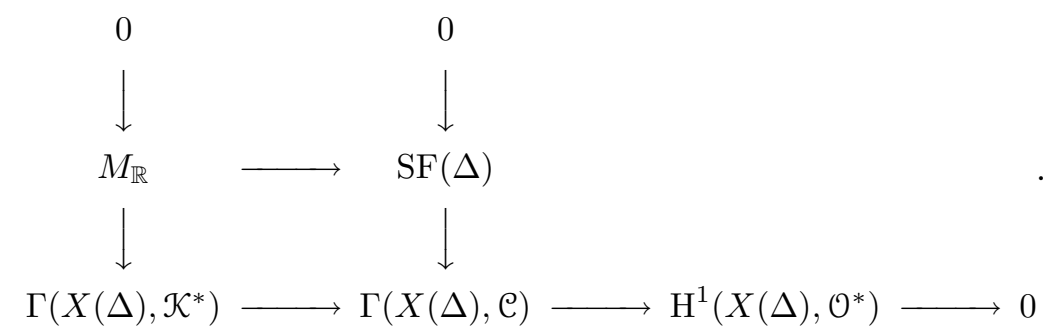

Proposition 4.10. Let $\Delta$ be a fan on $N_{\mathbb{R}}$. The diagram (4.5) above induces an exact sequence

$$
M_{\mathbb{R}} \rightarrow \mathrm{SF}(\Delta) \rightarrow \mathrm{H}^{1}\left(X(\Delta), \mathcal{O}^{*}\right) \rightarrow 0 .
$$

Suppose $\Delta$ is simplicial and $s=\operatorname{dim}_{\mathbb{R}} \mathbb{R}|\Delta|$. Then $\mathrm{H}^{1}\left(X(\Delta), \mathcal{O}^{*}\right) \cong \mathbb{R}^{\rho_{1}}$ where $\rho_{1}=$ $\#(\Delta(1))-s$.

Proof. As shown above, every Cartier divisor differs from an element of $\mathrm{SF}(\Delta)$ by a principal divisor, so the sequence (4.6) is exact. The kernel of $M_{\mathbb{R}} \rightarrow \mathrm{SF}(\Delta)$ consists of those linear functionals in $M_{\mathbb{R}}$ that vanish on $|\Delta|$. Because each $\sigma_{i} \in$ $\Delta(n)$ is simplicial, every linear functional $f_{i}$ on $\left|\sigma_{i}\right|$ is completely determined by its values on the one-dimensional faces of $\sigma_{i}$. There are exactly $n$ one-dimensional faces of $\sigma_{i}$. So $\operatorname{dim}_{\mathbb{R}} \operatorname{SF}(\Delta)=\#(\Delta(1))$.

Corollary 4.11. Let $\Delta$ be a rational fan on $N_{\mathbb{R}}$ and $X=T_{N} \operatorname{emb}(\Delta)$. Then

$$
\mathrm{H}^{1}\left(X(\Delta), \mathcal{O}^{*}\right) \cong \operatorname{Pic} X \otimes \mathbb{R} .
$$

Proof. Denote by $\operatorname{SF}(N, \Delta)$ the set of $\mathbb{Z}$-valued $\Delta$-linear support functions on $|\Delta|$. By $[13$, Corollary 2.5] there is an exact sequence

$$
M \rightarrow \mathrm{SF}(N, \Delta) \rightarrow \operatorname{Pic} X \rightarrow 0 .
$$

Since $\operatorname{SF}(N, \Delta) \otimes \mathbb{R}=\operatorname{SF}(\Delta)$, the result follows from Proposition 4.10.

Example 4.12. This example shows that Corollary 4.11 is false without the assumption that $\Delta$ is a rational fan. Let $\Delta$ be a complete fan on $N_{\mathbb{R}}=\mathbb{R}^{2}$ with exactly three one-dimensional cones, each being irrational. Then by Example 4.8, $\operatorname{Pic} X(\Delta) \cong \mathbb{R}$ and by Example 3.10, $\operatorname{Pic}\left(T_{N} \operatorname{emb}(\Delta)\right)=0$.

Corollary 4.13. Let $\Delta$ be a simplicial fan on $N_{\mathbb{R}}$ and $\Sigma=\Delta(1) \cup \Delta(0)$ the subfan consisting of all cones in $\Delta$ of dimension one or zero. Then

$$
\operatorname{Pic} X(\Delta)=\mathrm{H}^{1}\left(X(\Delta), \mathcal{O}^{*}\right) \cong \mathrm{H}^{1}\left(X(\Sigma), \mathcal{O}^{*}\right) \cong \mathbb{R}^{\rho_{1}}
$$

where $\rho_{1}=\#(\Delta(1))-s$, and $s=\operatorname{dim}_{\mathbb{R}} \mathbb{R}|\Delta|$. 
Proof. The natural map $\mathrm{SF}(\Delta) \rightarrow \mathrm{SF}(\Sigma)$ is an isomorphism, since $\Sigma$ contains all of the one-dimensional cones of $\Delta, \Delta$ is simplicial and $\Delta(1)=\Sigma(1)$. The rest follows from Proposition 4.10 and its proof.

Now suppose $\Delta$ is a fan on $N_{\mathbb{R}}$, where $N$ has rank $n$. Let $\left\{\sigma_{0}, \ldots, \sigma_{w}\right\}$ be a set of maximal cones in $\Delta$. For each $i$, suppose $\operatorname{dim} \sigma_{i} \geq 2$ and let $P_{i}$ denote the orbit corresponding to $\sigma_{i}$. Since the cones $\sigma_{i}$ are maximal, the sets $P_{i}$ are closed and pairwise disjoint. Let $P$ denote the union $P_{0} \cup \cdots \cup P_{w}$. Denote by $\Delta^{\prime}$ the fan obtained from $\Delta$ by deleting $\left\{\sigma_{0}, \ldots, \sigma_{w}\right\}$. Hence $\Delta^{\prime}=\Delta-\left\{\sigma_{0}, \ldots, \sigma_{w}\right\}$. Then $X\left(\Delta^{\prime}\right)=X(\Delta)-P$. Let $X_{i}=X_{\sigma_{i}}$ be the affine scheme Spec $k\left[\sigma_{i}^{\llcorner}\right]$. Then $X_{i}-P_{i}$ is the scheme associated to the fan $\Delta\left(\sigma_{i}\right)-\left\{\sigma_{i}\right\}$.

Proposition 4.14. In the above context, there is an exact sequence

$$
0 \rightarrow \mathrm{H}^{1}\left(X(\Delta), \mathcal{O}^{*}\right) \rightarrow \mathrm{H}^{1}\left(X\left(\Delta^{\prime}\right), \mathcal{O}^{*}\right) \rightarrow \coprod_{i=0}^{w} \mathrm{H}^{1}\left(X_{i}-P_{i}, \mathcal{O}^{*}\right)
$$

with natural maps.

Proof. Consider the cohomology groups with support in $P$. Because the $P_{i}$ are mutually disjoint, the cohomology with support in $P, \mathrm{H}_{P}^{j}\left(X(\Delta), \mathcal{O}^{*}\right)$, decomposes into a direct sum, $\bigsqcup_{i=0}^{w} \mathrm{H}_{P_{i}}^{j}\left(X(\Delta), \mathcal{O}^{*}\right)$. For each $i, \mathrm{H}_{P_{i}}^{j}\left(X(\Delta), \mathcal{O}^{*}\right)=\mathrm{H}_{P_{i}}^{j}\left(X_{i}, \mathcal{O}^{*}\right)$, by excision, since $X_{i}$ is an open neighborhood of $P_{i}$. The long exact sequence of the couple $(P, X(\Delta))$ is

$$
\cdots \rightarrow \mathrm{H}^{j}\left(X(\Delta), \mathcal{O}^{*}\right) \rightarrow \mathrm{H}^{j}\left(X\left(\Delta^{\prime}\right), \mathcal{O}^{*}\right) \rightarrow \coprod_{i=0}^{w} \mathrm{H}_{P_{i}}^{j+1}\left(X_{i}, \mathcal{O}^{*}\right) \rightarrow \cdots
$$

For each $i$ we also have the long exact sequence for the couple $\left(P_{i}, X_{i}\right)$.

$$
\cdots \rightarrow \mathrm{H}^{j}\left(X_{i}, \mathcal{O}^{*}\right) \rightarrow \mathrm{H}^{j}\left(X_{i}-P_{i}, \mathcal{O}^{*}\right) \rightarrow \mathrm{H}_{P_{i}}^{j+1}\left(X_{i}, \mathcal{O}^{*}\right) \rightarrow \cdots
$$

Consider the groups in degree $j=1$. By (4.6) since $M_{\mathbb{R}} \rightarrow \mathrm{SF}(\Delta)$ and $\mathrm{SF}(\Delta) \rightarrow$ $\mathrm{SF}\left(\Delta^{\prime}\right)$ are injective, $\mathrm{H}^{1}\left(X(\Delta), \mathcal{O}^{*}\right) \rightarrow \mathrm{H}^{1}\left(X\left(\Delta^{\prime}\right), \mathcal{O}^{*}\right)$ is injective. For each $i$, combine (4.8) and (4.9) to form the commutative square below.

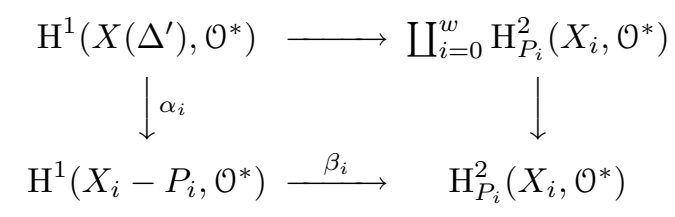

By Proposition 4.9, $\mathrm{H}^{1}\left(X_{i}, \mathcal{O}^{*}\right)=\operatorname{Pic} X_{i}=0$, hence $\beta_{i}$ is injective. Now (4.8) and (4.10) combine to give the exact sequence (4.7).

4.3. A conjecture on the Picard group for nonsimplicial fans. Following [5, Sec. 4] we now define an open neighborhood of a fan. Let $\Delta$ be a fan on $N \otimes \mathbb{R}=\mathbb{R}^{n}$ where $N$ has rank $n$. Let $\Delta(1)=\left\{r_{0}, \ldots, r_{m}\right\}$. The intersection of $\Delta(1)$ with the unit sphere $S$ in $\mathbb{R}^{n}$ is a finite set of points, say $\left\{p_{0}, \ldots, p_{m}\right\}$. About each $p_{i}$ we can find an open ball $B_{i}$ on $S$ such that if $p_{i}$ is parametrized by $B_{i}$, then each choice of $\vec{p}=\left(p_{0}, p_{1}, \ldots, p_{m}\right)$ in $B_{0} \times B_{1} \times \cdots \times B_{m}$ defines a fan $\Phi=\Phi(\vec{p})$. Under the topology defined on a fan in Section $1, \mathfrak{T}(\Phi)$ is homeomorphic to $\mathfrak{T}(\Delta)$, where $\mathfrak{T}$ is as in (1.1). Let $[\Delta]$ denote the homeomorphism class of the topological space $\Delta$. 


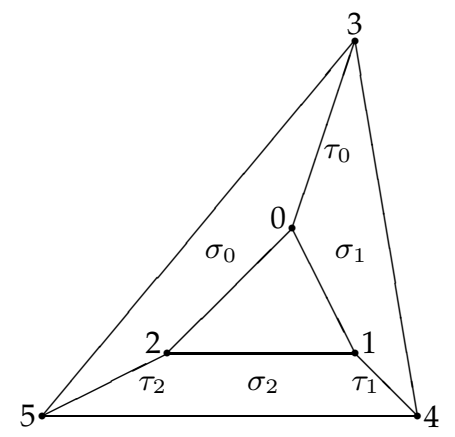

FIGURE 2

Thus the manifold $B=\prod_{i=0}^{m} B_{i}$ parametrizes a subset of fans in the fiber $\mathfrak{T}^{-1}([\Delta])$. Call $B$ an open neighborhood of $\Delta$.

As motivation for Conjecture 4.15 below, consider the following example in dimension three. Let $\Delta$ be a complete fan on $\mathbb{R}^{3}$ such that each $\sigma \in \Delta(3)$ has exactly four one-dimensional faces. Let $\Delta(3)=\left\{\sigma_{0}, \ldots, \sigma_{w}\right\}, \Delta(2)=\left\{\tau_{0}, \ldots, \tau_{e}\right\}$, $\Delta(1)=\left\{r_{0}, \ldots, r_{m}\right\}$. The intersections of the cones in $\Delta(2)$ with the unit sphere $S$ in $\mathbb{R}^{3}$ trace out the edges of a graph on $S$. This graph has $e+1$ edges, $m+1$ vertices and $w+1$ regions. So $w+1=(e+1)-(m+1)+2$. Each $\sigma_{j}$ has exactly four $\tau_{i}{ }^{\prime}$ s and each $\tau_{i}$ is in exactly two $\sigma_{j}$ 's, so $2(e+1)=4(w+1)$ or $e+1=2(w+1)$. Hence $w+1=m-1$. In (4.7), $\mathrm{H}^{1}\left(X\left(\Delta^{\prime}\right), \mathcal{O}^{*}\right)$ has dimension $(m+1)-3=m-2$ and for each $i, \mathrm{H}^{1}\left(X_{i}-P_{i}, \mathcal{O}^{*}\right)$ has dimension $4-3=1$. Therefore, in (4.7) the middle term has dimension $m-2$ and the third term dimension $m-1$. For each $i$, since $\mathrm{SF}\left(\Delta^{\prime}\right) \rightarrow \operatorname{SF}\left(\Delta\left(\sigma_{i}\right)-\left\{\sigma_{i}\right\}\right)$ is surjective, by (4.6), the natural map

$$
\alpha_{i}: \mathrm{H}^{1}\left(X\left(\Delta^{\prime}\right), \mathcal{O}^{*}\right) \rightarrow \mathrm{H}^{1}\left(X_{i}-P_{i}, \mathcal{O}^{*}\right)
$$

is surjective. So we can view $\mathrm{H}^{1}\left(X(\Delta), \mathcal{O}^{*}\right)$ as the intersection of $m-1$ hyperplanes through (0) in $\mathbb{R}^{m-2}$. In general, this intersection should be (0).

We restate in the context of $X(\Delta)$ a conjecture which was first made in the context of toric varieties.

Conjecture 4.15. [5, Conjecture 4.3] Let $\Delta$ be a complete fan on $\mathbb{R}^{3}$ such that for each cone $\sigma \in \Delta(3), \sigma$ is nonsimplicial. Let $B$ be an open neighborhood of $\Delta$ as described above. Then for a general choice of $\vec{p} \in B$, if $\Phi=\Phi(\vec{p})$, then every $\Phi$-linear support function is linear. In particular for a general choice of $\vec{p} \in B, \mathrm{H}^{1}\left(X(\Phi), \mathcal{O}^{*}\right)=0$.

Example 4.16. As in Example 4.8, we compute some Čech cohomology groups of $X(\Delta)$ but in this example $\Delta$ is a fan on $\mathbb{R}^{3}$. Suppose $\Delta$ consists of three cones of dimension three, nine cones of dimension two, six cones of dimension one and the cone of dimension zero. Assume that the intersection of the fan $\Delta$ with the unit sphere traces a graph that looks like that shown in Figure 2.

Suppose $\sigma_{i} \cap \sigma_{i+1}=\tau_{i}$ with subscripts interpreted $(\bmod 3)$. Then $X(\Delta)$ has an open cover $\mathcal{U}=\left\{X_{\sigma_{0}}, X_{\sigma_{1}}, X_{\sigma_{2}}\right\}$ and $X_{\sigma_{i}} \cap X_{\sigma_{i+1}}=X_{\tau_{i}}$. Also $X_{0} \cap X_{1} \cap$ $X_{2}=\operatorname{Spec} k\left[M_{\mathbb{R}}\right]$. We compute the cohomology groups $\check{H}^{i}\left(\mathcal{U}, \mathcal{O}^{*}\right)$ with respect to the sheaf of units $\mathcal{O}^{*}$ on $X(\Delta)$. It follows from Proposition $4.9 \check{\mathrm{H}}^{1}\left(\mathcal{U}, \mathcal{O}^{*}\right) \cong$ 
$\mathrm{H}^{1}\left(X(\Delta), \mathcal{O}^{*}\right)=\operatorname{Pic} X(\Delta)$. We have $\Gamma\left(X_{\sigma_{i}}, \mathcal{O}^{*}\right)=k^{*}$ for each $i$. Since $\operatorname{dim} \tau_{i}=2$,

$$
\Gamma\left(U_{\tau_{i}}, \mathcal{O}^{*}\right)=k^{*} \times \tau_{i}^{\perp} \cong k^{*} \times \mathbb{R}
$$

for each $i$. Also $\Gamma\left(k\left[M_{\mathbb{R}}\right], \mathcal{O}^{*}\right) \cong k^{*} \times M_{\mathbb{R}}$. The Čech complex for $\mathcal{U}$ is

$$
0 \rightarrow \bigoplus_{i=0}^{2} \Gamma\left(U_{\sigma_{i}}, \mathcal{O}^{*}\right) \stackrel{\delta^{0}}{\longrightarrow} \bigoplus_{i=0}^{2} \Gamma\left(U_{\tau_{i}}, \mathcal{O}^{*}\right) \stackrel{\delta^{1}}{\longrightarrow} \Gamma\left(k\left[M_{\mathbb{R}}\right], \mathcal{O}^{*}\right) \stackrel{\delta^{2}}{\longrightarrow} 0
$$

which becomes, after substitution,

$$
0 \rightarrow k^{*} \oplus k^{*} \oplus k^{*} \stackrel{\delta^{0}}{\longrightarrow} k^{*} \times \tau_{0}^{\perp} \oplus k^{*} \times \tau_{1}^{\perp} \oplus k^{*} \times \tau_{2}^{\perp} \stackrel{\delta^{1}}{\longrightarrow} k^{*} \times M_{\mathbb{R}} \stackrel{\delta^{2}}{\longrightarrow} 0 .
$$

Therefore

$$
\begin{gathered}
\check{\mathrm{H}}^{0}\left(\mathcal{U}, \mathcal{O}^{*}\right)=k^{*}, \\
\check{\mathrm{H}}^{1}\left(\mathcal{U}, \mathcal{O}^{*}\right) \cong\left(\tau_{0}^{\perp}+\tau_{1}^{\perp}+\tau_{2}^{\perp}\right)^{\perp}, \\
\check{\mathrm{H}}^{2}\left(\mathcal{U}, \mathcal{O}^{*}\right)=M_{\mathbb{R}} /\left(\tau_{0}^{\perp}+\tau_{1}^{\perp}+\tau_{2}^{\perp}\right) .
\end{gathered}
$$

For instance, for $\Delta$, take $\Delta(1)$ to be $\left\{\mathbb{R}_{\geq} \eta_{i} \mid i=0 . .5\right\}$ where $\left\{\eta_{0}, \ldots, \eta_{5}\right\}=$

$$
\left\{\left(\begin{array}{c}
1 \\
0 \\
-2
\end{array}\right),\left(\begin{array}{c}
-1 \\
2 \\
-2
\end{array}\right),\left(\begin{array}{l}
-1 \\
-2 \\
-2
\end{array}\right),\left(\begin{array}{l}
1 \\
0 \\
2
\end{array}\right),\left(\begin{array}{c}
-1 \\
2 \\
2
\end{array}\right),\left(\begin{array}{c}
-1 \\
-2 \\
2
\end{array}\right)\right\} .
$$

Check that $\check{\mathrm{H}}^{1}\left(\mathcal{U}, \mathcal{O}^{*}\right) \cong \mathbb{R}$ and $\check{\mathrm{H}}^{2}\left(\mathcal{U}, \mathcal{O}^{*}\right) \cong \mathbb{R}$. Next take $\Delta$ to be less symmetric. Take $\Delta(1)$ to be $\left\{\mathbb{R}_{\geq} \eta_{i} \mid i=0 . .5\right\}$ where $\left\{\eta_{0}, \ldots, \eta_{5}\right\}=$

$$
\left\{\left(\begin{array}{l}
0 \\
1 \\
1
\end{array}\right),\left(\begin{array}{l}
0 \\
0 \\
1
\end{array}\right),\left(\begin{array}{l}
1 \\
0 \\
1
\end{array}\right),\left(\begin{array}{c}
-1 \\
3 \\
1
\end{array}\right),\left(\begin{array}{c}
-2 \\
-1 \\
1
\end{array}\right),\left(\begin{array}{c}
3 \\
-1 \\
1
\end{array}\right)\right\} .
$$

Check that $\check{\mathrm{H}}^{1}\left(\mathcal{U}, \mathcal{O}^{*}\right)=0$ and $\check{\mathrm{H}}^{2}\left(\mathcal{U}, \mathcal{O}^{*}\right)=0$.

The next proposition shows that a counterexample to Conjecture 4.15 cannot be the fan associated to a hyperplane arrangement.

Proposition 4.17. If $\mathcal{A}$ is a real hyperplane arrangement in $V=N_{\mathbb{R}}$, and $\Phi=\Phi(\mathcal{A})$ is the fan on $V \times \mathbb{R}$ associated to $\mathcal{A}$, as defined in Section 2.4, then the Picard group $\mathrm{H}^{1}\left(X(\Phi), \mathcal{O}^{*}\right)$ is non-trivial.

Proof. As in the proof of Proposition 3.9, decompose $\Phi$ into $\Phi_{-} \cup \Phi_{+}$where $\Phi_{+}$ (resp. $\Phi_{-}$) consists of those cones $\sigma \in \Phi$ such that $x_{0}$ is non-negative (resp. nonpositive) as a function on $\sigma$. So $\Phi_{0}=\Phi_{-} \cap \Phi_{+}$is a fan on the hyperplane $H_{0}$ where $x_{0}=0$. Let $X=X(\Phi)$ and $X_{i}=X\left(\Phi_{i}\right)$, for $i=-,+, 0$. Then

$$
\mathrm{H}^{0}\left(X_{i}, \mathcal{O}^{*}\right)= \begin{cases}k^{*} & \text { if } i=-,+, \\ k^{*} \times \mathbb{R} & \text { if } i=0 .\end{cases}
$$

The Mayer-Vietoris sequence corresponding to the double cover $X=X_{-} \cup X_{+}$is (4.12)

$$
1 \rightarrow \mathrm{H}^{0}\left(X, \mathcal{O}^{*}\right) \rightarrow \mathrm{H}^{0}\left(X_{-}, \mathcal{O}^{*}\right) \oplus \mathrm{H}^{0}\left(X_{+}, \mathcal{O}^{*}\right) \rightarrow \mathrm{H}^{0}\left(X_{0}, \mathcal{O}^{*}\right) \rightarrow \mathrm{H}^{1}\left(X, \mathcal{O}^{*}\right) \rightarrow \cdots .
$$

Which becomes

$$
1 \rightarrow k^{*} \rightarrow k^{*} \oplus k^{*} \rightarrow k^{*} \oplus \mathbb{R} \rightarrow \mathrm{H}^{1}\left(X, \mathcal{O}^{*}\right) \rightarrow \cdots .
$$

Therefore $\operatorname{dim}_{\mathbb{R}} \mathrm{H}^{1}\left(X, \mathcal{O}^{*}\right)$ is at least one. 
Example 4.18. Let $\mathcal{A}$ be a hyperplane arrangement in $\mathbb{R}^{2}$ consisting of three lines in general position. Let $\Phi=\Phi(\mathcal{A})$ be the fan associated to $\mathcal{A}$. Let $X, X_{-}, X_{+}$and $X_{0}$ be as in the proof of Proposition 4.17. Since $\Phi_{0}$ is simplicial, $\operatorname{dim}_{\mathbb{R}} \mathrm{H}^{1}\left(X_{0}, \mathcal{O}^{*}\right)=4$, by Proposition 4.10. Using (4.6), one computes

$$
\operatorname{dim}_{\mathbb{R}} \mathrm{H}^{1}\left(X_{-}, \mathcal{O}^{*}\right)=6 \text { and } \operatorname{dim}_{\mathbb{R}} \mathrm{H}^{1}\left(X_{+}, \mathcal{O}^{*}\right)=6 .
$$

Now (4.12) becomes

$$
0 \rightarrow \mathbb{R} \rightarrow \mathrm{H}^{1}\left(X, \mathcal{O}^{*}\right) \rightarrow \mathrm{H}^{1}\left(X_{-}, \mathcal{O}^{*}\right) \oplus \mathrm{H}^{1}\left(X_{+}, \mathcal{O}^{*}\right) \rightarrow \mathrm{H}^{1}\left(X_{0}, \mathcal{O}^{*}\right) .
$$

Because the last arrow is a surjection, it follows that $\operatorname{dim}_{\mathbb{R}} \mathrm{H}^{1}\left(X, \mathcal{O}^{*}\right)=9$.

Example 4.19. The proof of Theorem 3.1 of [3] shows that for any complete fan of dimension three there exists a rational fan $\Sigma$ of the same combinatorial type as $\Delta$ such that $X=T_{N} \operatorname{emb}(\Sigma)$ is projective and thus Pic $X \neq 0$. By Corollary 4.11, it follows that $\mathrm{H}^{1}\left(X(\Sigma), \mathcal{O}^{*}\right) \neq 0$. We say that two fans $\Delta$ and $\Sigma$ are of the same combinatorial type if the two partially ordered sets formed by the cones in the two fans are isomorphic. As is easy to see, $\Delta$ and $\Sigma$ are of the same combinatorial type if and only if $\Delta$ and $\Sigma$ are homeomorphic as topological spaces. Therefore $\Sigma$ is in $\mathfrak{T}^{-1}([\Delta])$. Can $\Sigma$ be chosen from within any open neighborhood $B$ of $\Delta$ ?

4.4. The Brauer group of $X(\Delta)$. In this section, if $X$ is a scheme, the Brauer group $\mathrm{B}(X)$ of classes of Azumaya $\mathcal{O}_{X}$-algebras is as defined in [8, I]. An algebra $A$ on $X$ is an Azumaya $\mathcal{O}_{X}$-algebra if $A$ is locally free of finite-type as an $\mathcal{O}_{X}$-module and the canonical homomorphism $A \otimes_{\mathcal{O}_{X}} A^{\circ} \rightarrow \operatorname{Hom}_{\mathcal{O}_{X}-\bmod }(A, A)$ is an isomorphism. We also will be using the notation established in the three paragraphs preceding Proposition 4.9.

Proposition 4.20. Let $\sigma$ be a strictly convex polyhedral cone in $N_{\mathbb{R}}$ and $\sigma$ the dual cone

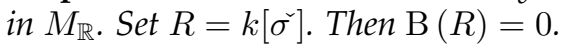

Proof. Let $A$ be an Azumaya $R$-algebra. As in the proof of Proposition 4.9, there is a finitely generated subsemigroup $\Omega$ of $\sigma$ such that if $S=k[\Omega]$, then there is an Azumaya $S$-algebra $A_{0}$ and $A \cong A_{0} \otimes_{S} R$. Furthermore, we can pick $S$ so that $S$ is the affine coordinate ring of an affine toric variety. Let $\overline{M_{0}}$ be the maximal subgroup of $\Omega$. Because $\overline{M_{0}}$ is finitely generated we can assume without loss of generality that $\overline{M_{0}} \subseteq \sigma^{\perp}$. Let $\bar{M}$ be the subgroup of $M_{\mathbb{R}}$ spanned by $\Omega$. By [2, Corollary 10], $\mathrm{B}(S) \rightarrow \mathrm{B}(k[\bar{M}])$ and $\mathrm{B}\left(k\left[\overline{M_{0}}\right]\right) \rightarrow \mathrm{B}(k[\bar{M}])$ are both injective and have the same image. So we can view $A_{0}$ as a representative of a class in $\mathrm{B}\left(k\left[\overline{M_{0}}\right]\right)$. So $A_{0}$ is Brauer equivalent to a product of symbols of the form $\left(\chi^{x}, \chi^{y}\right)_{\nu}$ where $x$, $y$ are in $\overline{M_{0}}$. But the symbol $\left(\chi^{x}, \chi^{y}\right)_{\nu}$ is split by $k\left[\overline{M_{0}}+\mathbb{Z} \cdot x / \nu+\mathbb{Z} \cdot y / \nu\right]$. Since $\{x, y\} \subseteq \overline{M_{0}} \subseteq \sigma^{\perp}$ it follows that $\{x / \nu, y / \nu\} \subseteq \sigma^{\perp} \subseteq \sigma$. Therefore, by adding at most a finite number of new generators to $\Omega$ we can assume that $A_{0}$ is split. So $A$ is split.

Theorem 4.21. Let $\Delta$ be a fan on $N_{\mathbb{R}}$. Then $\mathrm{B}(X(\Delta))=0$.

Proof. Let $A$ denote an Azumaya algebra on $X(\Delta)$ of rank $n^{2}$. The isomorphism classes of Azumaya algebras of rank $n^{2}$ over $X(\Delta)$ are parametrized by the set $\check{\mathrm{H}}^{1}\left(X(\Delta)_{\text {ét }}, \mathrm{PGL}_{n}\right)$. By Proposition $4.20 A$ is split by the open cover $\mathcal{U}=\left\{X_{\sigma}\right\}_{\sigma \in \Delta}$. Therefore $A$ corresponds to a 1-cocycle $y$ for $\mathrm{PGL}_{n}$ and the open cover $\mathcal{U}$. For each $\sigma$ in $\Delta$ there is a finitely generated semigroup $\Omega_{\sigma}$ in $\sigma$ such that on the open set 
$X_{\sigma}, y$ is defined over the subring $k\left[\Omega_{\sigma}\right] \subseteq k[\tilde{\sigma}]$. Suppose that for each $\sigma$ in $\Delta$ we have such an $\Omega_{\sigma}$. Also assume $\Omega_{\sigma}$ is integrally closed in the $\mathbb{Z}$-submodule of $M_{\mathbb{R}}$ spanned by $\Omega_{\sigma}$. Let $\bar{M}$ be the $\mathbb{Z}$-submodule of $M_{\mathbb{R}}$ spanned by $\bigcup_{\sigma \in \Delta} \Omega_{\sigma}$. Since each $\sigma$ contains a $\mathbb{Z}$-spanning set for $M_{\mathbb{R}}$, if necessary we can enlarge each $\Omega_{\sigma}$ so that $\Omega_{\sigma}$ is finitely generated as a semigroup, is integrally closed in $\bar{M}$ and spans $\bar{M}$ as a $\mathbb{Z}$-module. Given $\sigma, \tau$ in $\Delta$, let $\mu=\sigma \cap \tau$. If necessary, enlarge $\Omega_{\mu}$ so that $\Omega_{\mu}=\Omega_{\sigma}+\Omega_{\tau}$. This is possible since $\check{\mu}=\check{\sigma}+\tau \check{\tau}$ by Proposition 4.3. Set $\bar{N}=\operatorname{Hom}_{\mathbb{Z}}(\bar{M}, \mathbb{Z})$. For every $\sigma$ in $\Delta$, set $\bar{\sigma}=\left\{x \in \bar{N}_{\mathbb{R}} \mid\langle x, \omega\rangle \geq 0\right.$ for each $\left.\omega \in \Omega_{\sigma}\right\}$. Then $\bar{\sigma}$ is a strictly convex rational polyhedral cone in $\bar{N}_{\mathbb{R}}$ and $\left.\Omega_{\sigma}=\bar{\sigma}\right\urcorner \bar{M}$. Given that $\Omega_{\mu}=\Omega_{\sigma}+\Omega_{\tau}$, one can easily check that $\bar{\mu}=\bar{\sigma} \cap \bar{\tau}$ in $\bar{N}_{\mathbb{R}}$. Set $\bar{\Delta}=\bigcup_{\sigma \in \Delta} \Delta(\bar{\sigma})$, the set of all faces of cones $\bar{\sigma}$. The maximal cones in $\bar{\Delta}$ are of the form $\bar{\sigma}$, for some $\sigma$ in $\Delta$. Therefore, given 2 cones $\tau_{1}, \tau_{2}$ in $\bar{\Delta}$ there are cones $\sigma_{1}, \sigma_{2}$ in $\Delta$ such that $\tau_{1} \leq \overline{\sigma_{1}}$ and $\tau_{2} \leq \overline{\sigma_{2}}$. So $\tau_{1} \cap \tau_{2}$ is a face of $\overline{\sigma_{1}} \cap \overline{\sigma_{2}}=\overline{\sigma_{1} \cap \sigma_{2}}$; hence $\tau_{1} \cap \tau_{2}$ is in $\bar{\Delta}$. This shows that $\bar{\Delta}$ is a fan on $\bar{N}_{\mathbb{R}}$. The toric variety $T_{\bar{N}} \operatorname{emb}(\bar{\Delta})$ has the open affine

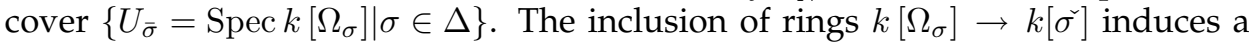
morphism of affine schemes $\phi_{\sigma}: X_{\sigma} \rightarrow U_{\bar{\sigma}}$ for each $\sigma$ in $\Delta$. Gluing the morphisms $\left\{\phi_{\sigma}\right\}_{\sigma \in \Delta}$ yields a morphism of schemes $\phi: X(\Delta) \rightarrow T_{\bar{N}} \operatorname{emb}(\bar{\Delta})$. In this way, the 1-cocycle $y$ corresponding to $A$ descends to a toric variety of the form $T_{\bar{N}} \operatorname{emb}(\bar{\Delta})$. Let $\bar{A}$ denote the Azumaya algebra over $\bar{X}=T_{\bar{N}} \operatorname{emb}(\bar{\Delta})$ defined by $y$ with respect to the sheaf $\mathrm{PGL}_{n}$ and the open cover $\left\{U_{\bar{\sigma}}\right\}_{\sigma \in \Delta}$. If $\phi: X(\Delta) \rightarrow T_{\bar{N}} \operatorname{emb}(\bar{\Delta})$ is as above, then $\phi^{*}(\bar{A})$ is Brauer equivalent to $A$. Since $\bar{A}$ is split by the open cover $\left\{U_{\bar{\sigma}}\right\}_{\sigma \in \Delta}$, by $[4$, Section 1$], \bar{A}$ represents a class in $\mathrm{B}(\bar{K} / \bar{X})$ where $\bar{K}$ is the function field of $\bar{X}$. By [2, Theorem 1] $\mathrm{H}^{1}(\mathfrak{T}(\bar{\Delta}), \mathcal{S F}) \cong \mathrm{H}^{2}\left(\bar{K} / \bar{X}_{\text {ét }}, \mathbb{G}_{m}\right)$. Since $\mathrm{B}(\bar{K} / \bar{X})$ embeds into the torsion subgroup of $\mathrm{H}^{2}\left(\bar{K} / \bar{X}_{\text {ét }}, \mathbb{G}_{m}\right)$ by a natural map [12, Theorem IV.2.5], $\bar{A}$ is represented by a 1 -cocycle on $\mathfrak{T}(\bar{\Delta})$ for the sheaf of $\mathbb{Z}$ valued $\bar{\Delta}$-linear support functions $\mathcal{S F}$. Torsion in $\mathrm{H}^{1}(\mathfrak{T}(\bar{\Delta}), \mathcal{S F})$ of order $n$ can be split by the morphism $T_{n \bar{N}} \operatorname{emb}(\bar{\Delta}) \rightarrow T_{\bar{N}} \operatorname{emb}(\bar{\Delta})$ of toric varieties induced by the submodule $n \bar{N} \subseteq \bar{N}$. By replacing $\bar{M}$ with $n \bar{M}$ if necessary, we can assume that the algebra $\bar{A}$ is split. Therefore $A$ is split.

\section{ACKNOWLEDGMENT}

The author wishes to thank Bernard Johnston for several helpful discussions on this material.

\section{REFERENCES}

[1] D. Cox, The homogeneous coordinate ring of a toric variety, J. Algebraic Geom. 4 (1995), 17-50.

[2] F. R. DeMeyer, T. J. Ford, and R. Miranda, The cohomological Brauer group of a toric variety, J. Algebraic Geom. 2 (1993), 137-154.

[3] M. Eikelberg, Picard groups of compact toric varieties and combinatorial classes of fans, Resultate Math. 23 (1993), 251-293.

[4] T. J. Ford, Examples of locally trivial Azumaya algebras, $K$-theory and Algegraic Geometry: Connections with Quadratic Forms and Division Algebras (Santa Barbara, CA, 1992), Proceedings of Symposia in Pure Mathematics, vol. 58, Amer. Math. Soc., Providence, RI, 1995, pp. 197-216.

[5] _ Topological invariants of a fan associated to a toric variety, Comm. Algebra 23 (1995), 40314045 .

[6] William Fulton, Introduction to toric varieties, Annals of Mathematics Studies, vol. 131, Princeton University Press, Princeton, New Jersey, 1993.

[7] R. Gilmer, Commutative semigroup rings, Chicago Lectures in Mathematics, The University of Chicago Press, Chicago and London, 1984. 
[8] A. Grothendieck, Le groupe de Brauer I, II, III, Dix Exposés sur la Cohomologie des Schémas, North Holland, Amsterdam, 1968, pp. 46-188.

[9] R. Hartshorne, Algebraic geometry, Graduate Texts in Mathematics, vol. 52, Springer-Verlag, New York/Berlin, 1977.

[10] G. Karpilovsky, Commutative group algebras, Pure and Applied Mathematics, vol. 78, Marcel Dekker, New York/Basel, 1983.

[11] U. Krause, On monoids of finite real character, Proc. Amer. Math. Soc. 105 (1989), 546-554.

[12] J. Milne, Etale cohomology, Princeton Mathematical Series, vol. 33, Princeton University Press, Princeton, N.J., 1980.

[13] T. Oda, Convex bodies and algebraic geometry, Ergebnisse der Mathematik und ihrer Grenzgebiete, 3. Folge, vol. 15, Springer-Verlag, Berlin/Heidelberg, 1988.

[14] P. Orlik and Hiroaki Terao, Arrangements of hyperplanes, Grundlehren der mathematischen Wissenschaften, vol. 300, Springer-Verlag, Berlin/Heidelberg, 1992.

[15] O. Zariski and P. Samuel, Commutative algebra, I and II, Graduate Texts in Mathematics, vol. 28 and 29, Springer-Verlag, New York/Heidelberg, 1960.

Department of Mathematics, Florida Atlantic University, Boca RAton, Florida 33431

E-mail address: Fordefau.edu, URL: http://www.math.fau.edu/ford/ 